\title{
Optimal alternative for suitability of $S$-boxes to image encryption based on $m$-polar fuzzy soft set decision-making criterion
}

\author{
Mohammed M. Khalaf
}

Correspondence:

Khalfmohammed2003@yahoo.com

Higher Institute of Engineering and Technology King Marriott, P.O. Box

3135, Alexandria, Egypt

\begin{abstract}
Our aim in this work is to improve the design and model of real-life applications. We put forward a standard based on m-polar fuzzy soft set decision-making criterion to examine the optimal alternative for the suitability of S-boxes to image encryption applications. The proposed standard studies the results of correlation analysis, entropy analysis, contrast analysis, homogeneity analysis, energy analysis, and mean of absolute deviation analysis. These analyses are applied to well-known substitution boxes. The algorithm of outcomes of these analyses is additional observed and a $m$-polar fuzzy soft set decision-making criterion is used to decide the optimal alternative for suitability of S-box to image encryption applications. All results taken by using the reality values for all S-boxes and experimental problems with reality values are discussed to show the validity of the optimal alternative for the suitability of S-box to image encryption.
\end{abstract}

Keywords: $8 \times 8$ S-boxes, Image encryption, Homogeneity analysis, S-boxes, Decisionmaking criterion

AMS Classification: $03 \mathrm{E} 72,47540$

\section{Introduction}

The block ciphers (symmetric key cryptosystem) present an essential job in the area of secure communications. The security of an encryption algorithm is related to the performance of the building block which is liable for producing uncertainty in the cipher. This functionality is attained by the use of an S-box, so this component is like a nucleus in an atom [1]. The perfection in the properties of an S-boxes has been a major problem of research in the area of cryptology. In this paper, we show the correlation analysis, entropy analysis, contrast analysis, homogeneity analysis, energy analysis, and mean of absolute deviation analysis for existing S-boxes. The correlation analysis is widely used to analyses the S-box's statistical properties [2]. The entropy analysis is a statistical method used to measure the uncertainty in image data. The amount of uncertainty in an encrypted image characterizes the texture of the image. In contrast analysis [3], the intensity difference between a pixel and its neighbor over the whole image is calculated. The elevated values of contrast analysis reflect the amount of randomness in encrypted images and results in enhanced security. The measure of closeness in the

(c) The Author(s). 2020 Open Access This article is distributed under the terms of the Creative Commons Attribution 4.0 International License (http://creativecommons.org/licenses/by/4.0/), which permits unrestricted use, distribution, and reproduction in any medium, provided you give appropriate credit to the original author(s) and the source, provide a link to the Creative Commons license, and indicate if changes were made. 
distribution of grey-level co-occurrence matrix (GLCM) elements to the GLCM diagonal is calculated by the use of homogeneity analysis [4]. The GLCM is the tabulation of how often different combinations of pixel brightness values (grey levels) occur in an image [5]. In another method, energy analyzes the sum of squared elements in the GLCM. This analysis provides the merits and demerits of various S-boxes in terms of energy of the resulting encrypted image. The final method that we implement on the encrypted image is the mean of absolute deviation (MAD) analysis [6]. This analysis determines the difference in the original and an encrypted image. There are numerous emerging encryption methods recently proposed in the literature. Although these algorithms appear to be promising, their robustness is not yet established and they are evolving to become standards. Some of these algorithms worth mentioning are the public-key cryptosystems based on chaotic Chebyshev polynomials [7], the advanced encryption standard (AES) cryptosystem using the features of mosaic image for extremely secure high data rate [8], and image encryption via logistic map function and heap tree [9]. The most common methods used to analyze the statistical strength of Sboxes are the correlation analysis, linear approximation probability, differential approximation probability, strict avalanche criterion, etc. We have included the correlation method as a benchmark for the remaining analysis used in this work. With the exception of correlation analysis, the application and use of the results of statistical analysis, presented in this paper, have not been applied to evaluate the strength of S-boxes. The correlation analysis, entropy analysis, contrast analysis, homogeneity analysis, energy analysis, and mean of absolute deviation analysis are performed on AES [10], APA [11], Gray [1], Lui J [12], residue prime [13], S8 AES [14], SKIPJACK [15], and Xyi [16] Sboxes. The results of these analyses are studied by the proposed criterion, and a fuzzy soft set decision is reached by taking into account the values of all the analysis on the different S-boxes. On the other hand, Majumdar and Samanta [17] presented the concept of generalized fuzzy soft sets, followed by studies on generalized multi-fuzzy soft sets [18], generalized intuitionistic fuzzy soft sets [19, 20], generalized fuzzy soft expert set [21], and generalized interval-valued fuzzy soft set [22]. Recently, Zhu and Zhan [23] proposed the concept of fuzzy parameterized fuzzy soft sets, along with decisionmaking. Zhao et al. [24] presented a novel decision-making approach based on intuitionistic fuzzy soft sets. Deli [25] introduced the notion of interval-valued neutrosophic soft sets and its decision-making. Fatimah et al. [26, 27] extended models include Nsoft sets, and hybrid models include interval-valued fuzzy soft sets and (dual) probabilistic soft sets. In view of these developments, we will highlight the notion of possibility $m$-polar fuzzy soft set, which can be seen as a new possibility $m$-polar fuzzy soft model. Figures 1 and 2 explain the image before and after encryption.

\section{Preliminaries and basic definitions}

Wang et al. [28] provided the set-theoretic operators and various properties of SVNSs. Ye $[29,30]$ proposed a multi-attribute decision-making (MADM) method using the correlation coefficient under single-valued neutrosophic environment. Ye [31, 32] further developed clustering method and decision-making methods by similarity measures of SVNS. Meanwhile, Peng and Dai [33] presented a new similarity measure of SVNS and applied them to decision-making. Biswas et al. [34] extended the Technique for Order Preference by Similarity to an Ideal Solution (TOPSIS) method for multi- 


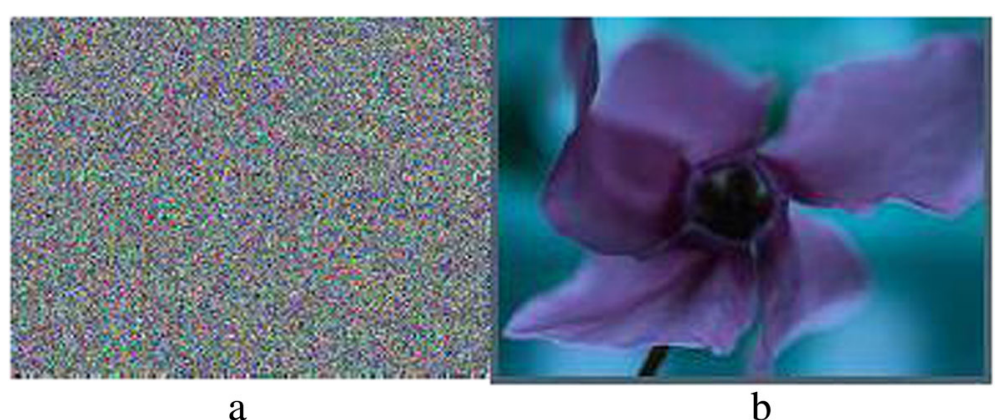

Fig. 1 The image before and after encryption. This figure explained the difference between after and before encryption

attribute single-valued neutrosophic decision-making problem. Sahin and Kucuk [35] defined a subsethood measure for SVNS and applied to MADM. Evaluation based on distance from average solution (EDAS), originally proposed by Ghorabaee et al. [36], is a new MADM method for inventory ABC classification. It is very useful when we have some conflicting parameters. In the compromise MADM methods such as TOPSIS and VIKOR [37], the best alternative is got by computing the distance from ideal and nadir solutions. The desirable alternative has lower distance from ideal solution and higher distance from nadir solution in these MADM methods. Ghorabaee et al. [38] extended the EDAS method to supplier selection. As far as we know, however, the study of the MADM problem based on EDAS method has not been reported in the existing academic literature. Hence, it is an interesting research topic to apply the EDAS in MADM to rank and determine the best alternative under the single-valued neutrosophic soft environment. Through a comparative analysis of the given methods, their objective valuation is carried out, and the method which maintains consistency of its results is chosen. For computing the similarity measure of two SVNSs, we propose a new axiomatic definition of the similarity measure, which takes in the form of SVNN. Comparing with the existing literature $[31,32,39,40]$, our similarity measure can remain more original decision information. By means of level soft sets, Feng et al. [41] presented an adjustable approach to fuzzy soft sets based decision-making. By considering different

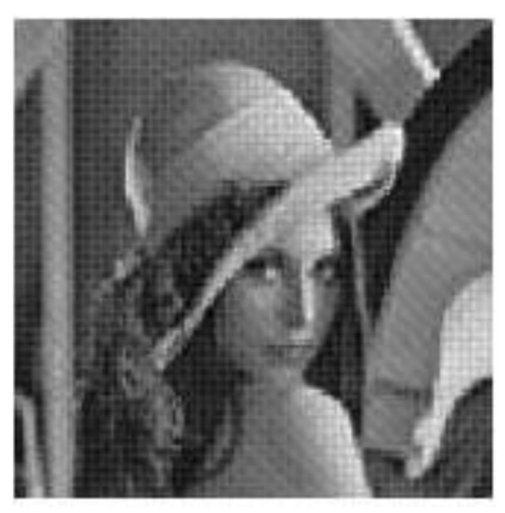

a

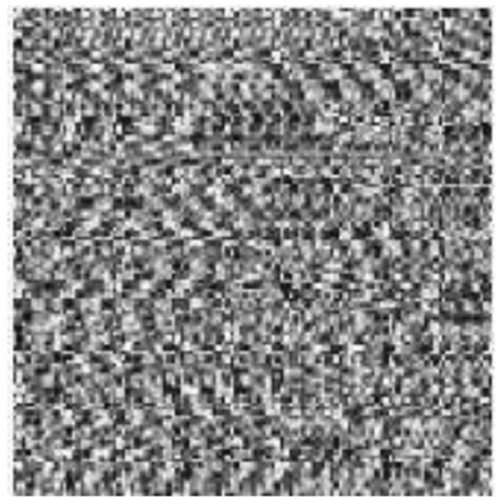

b

Fig. 2 The image before and after encryption. This figure explained the difference between after and before encryption 
types of thresholds, it can derive different level soft sets from the original fuzzy soft set. In general, the final optimal decisions based on different level soft sets could be different. Thus, the newly proposed approach is, in fact, an adjustable method which captures an important feature for decision-making in an imprecise environment: some of these problems are essentially humanistic and thus subjective in nature. As far as we know, however, the study of the single-valued neutrosophic soft MADM problem based on level soft set has not been reported in the existing academic literature. Considering that different attribute weights will influence the ranking results of alternatives, we develop a new method to determine the attribute weights by combining the subjective elements with the objective ones. This model is different from the existing methods, which can be divided into two tactics: one is the subjective weighting evaluation methods and the other is the objective weighting determine methods, which can be computed by grey system theory [42]. Figures 1 and 2 explain the image before and after encryption. For more information about $m$-polar fuzzy sets and analyses of S-Box in image encryption applications based on fuzzy decision-making criterion.

The remainder of this paper is organized as follows: firstly, Sections 1 and 2 introduced some background of image encryption, showed and analyzed the types of the Sboxes. AES, APA, Gray, Lui J, residue prime, S8 AES, SKIPJAC, and Xyi talked about the properties of these S-boxes (the correlation analysis, entropy analysis, contrast analysis, homogeneity analysis, energy analysis, and mean of absolute deviation). Also, these sections explained soft set, fuzzy soft set, and fuzzy polar soft set.

In Section 4, the analyses of S-Box in image encryption applications based on fuzzy sets and 2-polar fuzzy soft set decision-making criterion are studied, in problem statement chosen suitability of S-box to image encryption based on polar fuzzy soft set and construct an algorithm for a decision-making.

In Section 4.2, we developed a study to state decision-making based on 2-polar fuzzy soft set by using two measures. Also, in Section 4.2, the optimal alternative for the suitability of S-box to image encryption based on 2-polar fuzzy soft set and by using a join and meet for 2-polar fuzzy soft set are introduced. All the results are taken by using the reality values for all S-boxes, and experimental examples with reality values are discussed to show the validity of the proposed concept.

Section 5 is the conclusion and remarks.

\section{Soft sets and $\boldsymbol{m}$-polar fuzzy soft set}

Let $E$ be a non-empty finite set of attributes (parameters, characteristics, or properties) which the objects in $U$ possess and let $P(U)$ denote the family of all subsets of $U$. Then a soft set is defined with the help of a set-valued mapping as given below:

Definition 2.1 (Molodtsov [43] A pair $(F, A)$ is called a soft set over $U$, where $A \subseteq E$ and $F: A \rightarrow P(U)$ is a set-valued mapping. In other words, a soft set $(F, A)$ over $U$ is a parameterized family of subsets of $U$ where each parameter $e \in A$ is associated with a subset $F(e)$ of $U$. The set $F(i)$ contains the objects of $U$ having the property $i$ and is called the set of $i$-approximate elements in $(F, A)$.

Definition 2.2 (Chen, Li and Koczy, $[44,45])$ Elements $\left([0,1]^{m}\right)^{X}$ the set of all mappings from $X$ to $[0,1]^{m}$ with the point - wise order are called an $m$-polar fuzzy sets, such that $m$ is an arbitrary cardinality. A subset $\mathcal{A}=\left\{\mathcal{A}_{k}\right\}_{k \in K} \subseteq\left([0,1]^{m}\right)^{X}$ (or a mapping $\mathcal{A}: K \rightarrow\left([0,1]^{m}\right)^{X}$ satisfying $\left.\mathcal{A}(k)=\mathcal{A}_{k} \quad \forall k \in K\right)$ is called an an $m$-polar fuzzy soft set on $X$. 
Example 2.1 Let $X=\left\{a_{1}, a_{2}\right\}$ be a two element set, $I=\left\{i_{1}, i_{2}, i_{3}\right\}$ be a four-element set, the 2-polar fuzzy soft set $\mathcal{A} \in\left[\left([0,1]^{2}\right)^{X} \times\left([0,1]^{2}\right)^{X}\right]^{I}$ defined by:

$$
\begin{aligned}
& \mathcal{A}\left(a_{1}\right)=\left\{\frac{(0.6733,0.4325)}{i_{1}}, \frac{(0.2455,0.1985)}{i_{2}}, \frac{(0.8771,0.4765)}{i_{3}}\right\} \\
& \mathcal{A}\left(a_{2}\right)=\left\{\frac{(0.9325,0.6325)}{i_{1}}, \frac{(0.7342,0.5675)}{i_{2}}, \frac{(0.0815,0.0421)}{i_{3}}\right\}
\end{aligned}
$$

Definition 2.3 Let $\left\{\mathcal{A}_{k}\right\}_{k \in K} \in\left[\left([0,1]^{m}\right)^{X}\right]^{I_{k}}$. Define m-polar fuzzy soft sets $\vee\left\{\mathcal{A}_{k}\right\}_{k \in K}=\max \left\{\mathcal{A}_{k}\right\}_{k \in K}$ and $\wedge\left\{\mathcal{A}_{k}\right\}_{k \in K}=\min \left\{\mathcal{A}_{k}\right\}_{k \in K}$.

\section{Analyses of S-box in image encryption applications based on fuzzy sets and 2-polar fuzzy soft set decision-making criterion}

\section{Proposed methodology and implementation}

We chose the $\mathrm{n} \times \mathrm{n}$ S-boxes (AES, APA, Gray, Lui J, Residue Prime, S8 AES, SKIPJACK, and Xyi) used in popular block ciphers to do analysis. $(n=2,3,4,5 \ldots)$. (In Table 1 and figure. The reality values Entropy, contrast, average eneragy, eneragy, homogeneity, mad of prevailing S-box are explained ).

Our aim is to examine the optimal alternative for suitability of S-boxes to image encryption. Correlation information plays the main role in stating the similarity of pixel patterns in the given image and its encrypted version by the use of techniques such as entropy analysis, contrast analysis, homogeneity analysis, energy analysis, and mean of absolute deviation analysis on the image. Now, we want to use the concept of soft set to choose the best S-box, so assume that we analyze S-boxes (AES, APA, Gray, Lui J, Residue, S8 AES, SKIPJACK, and Xyi ). There are form the set $X=\left\{x_{1}, x_{2}, x_{3}, x_{4}, x_{5}, x_{6}\right.$, $\left.x_{7}, x_{8}, x_{9}\right\}$ of alternatives where $x_{i}\{i=1,2,3,4,5,6,7,8,9)$ are S-boxes and the alternatives $x_{i}$. To evaluate the S-boxes, we take the parameters $I=\left\{i_{1}, i_{2}, i_{3}, i_{4}, i_{5}, i_{6}\right\}$ where $i_{1}$ stands for entropy, $i_{2}$ stands for contrast, $i_{3}$ stands for average correlation, $i_{4}$ stands for energy, $i_{5}$ stands for homogeneity, $i_{6}$ stands for MAD. These parameters are important with degree. (In Table 2 and Fig. 2 we explained the important values of Entropy, contrast, average eneragy, eneragy, homogeneity, mad of prevailing S-box).

Table 1 Entropy, contrast, average energy, energy, homogeneity, MAD of prevailing S-box

\begin{tabular}{lllllll}
\hline Image & Entropy & Contrast & Average energy & Energy & Homogeneity & MAD \\
\hline Plain image & 6.6733 & 0.2455 & 0.8771 & 0.2917 & 0.9334 & NA \\
AES & 7.9325 & 7.224 & 0.0815 & 0.0211 & 0.4701 & 43.544 \\
APA & 7.8183 & 8.9114 & 0.1258 & 0.0193 & 0.4665 & 62.066 \\
Liu J & 7.9325 & 7.224 & 0.1311 & 0.0211 & 0.4701 & 43.456 \\
Prime & 7.8811 & 6.9646 & 0.2769 & 0.0198 & 0.4728 & 53.089 \\
S8 & 7.9447 & 8.1274 & 0.0734 & 0.019 & 0.4552 & 58.389 \\
Gray & 7.9299 & 7.7961 & 0.1014 & 0.0198 & 0.4567 & 49.723 \\
Xyi & 7.9127 & 7.8942 & 0.1413 & 0.0188 & 0.4605 & 57.238 \\
SKIPJACK & 7.9839 & 5.4255 & 0.3123 & 0.0232 & 0.5004 & 52.733 \\
\hline
\end{tabular}


Table 2 The important values of entropy, contrast, average energy, energy, homogeneity, MAD of prevailing S-box

\begin{tabular}{llllll}
\hline Entropy & Contrast & Average energy & Energy & Homogeneity & MAD \\
\hline 8.00 & 1.00 & 1.00 & 1.00 & 1.00 & 60.00 \\
\hline
\end{tabular}

The data provided by the committee for decision-making use is the following 3-polar fuzzy soft set $\mathcal{A} \in\left[\left([0,60]^{3}\right)^{I}\right]^{X}=\left[\left([0,60]^{3}\right)^{X^{I}}\right]^{I}=\left([0,60]^{3}\right)^{I \times X}=\left([0,60]^{3}\right)^{X \times I}$ defined by:

$$
\begin{aligned}
& \mathcal{A}\left(x_{1}\right)=\left\{\begin{array}{l}
\frac{(6.6733,6.4325,6.7325)}{i_{1}}, \frac{(0.2455,0.1985,0.1987)}{i_{2}}, \frac{(0.8771,0.4765,0.9654)}{i_{3}}, \\
\frac{(0.2917,0.1986,0.2786)}{i_{4}}, \frac{(0.9334,0.2672,0.1198)}{i_{5}}, \frac{(0.000 .0 .000 .0 .0000)}{i_{6}}
\end{array}\right\} \\
& \mathcal{A}\left(x_{2}\right)=\left\{\begin{array}{l}
\frac{(7.9325,7.6325,6.9325)}{i_{1}}, \frac{(0.7342,0.5675,0.3891)}{i_{2}}, \frac{(0.0815,0.0421,0.0123)}{i_{3}}, \\
\frac{(0.0211,0.0165,0.0298)}{i_{4}}, \frac{(0.4701,0.2560,0.1786)}{i_{5}}, \frac{(43.544,53.224,33.944)}{i_{6}}
\end{array}\right\}
\end{aligned}
$$$$
\mathcal{A}\left(x_{3}\right)=\left\{\begin{array}{l}
\frac{(7.8183,7.4325,7.7325)}{i_{1}}, \frac{(0.8376,0.6210,0.7098)}{i_{2}}, \frac{(0.1258,0.4987,0.1987)}{i_{3}}, \\
\frac{(0.0193,0.0221,0.0232)}{i_{4}}, \frac{(0.4665,0.2983,0.1923)}{i_{5}}, \frac{(62.066,53.224,33.944)}{i_{6}}
\end{array}\right\}
$$$$
\mathcal{A}\left(x_{4}\right)=\left\{\begin{array}{l}
\frac{(7.9325,7.7325,7.5325)}{i_{1}}, \frac{(0.7210,0.8876,0.9129)}{i_{2}}, \frac{(0.1311,0.4943,0.1786)}{i_{3}}, \\
\frac{(0.0211,0.0131,0.0292)}{i_{4}}, \frac{(0.4701,0.2987,0.1876)}{i_{5}}, \frac{(43.456,53.224,33.944)}{i_{6}}
\end{array}\right\}
$$$$
\mathcal{A}\left(x_{5}\right)=\left\{\begin{array}{l}
\frac{(7.8811,7.6811,7.4811)}{i_{1}}, \frac{(0.6657,0.7987,0.5012)}{i_{2}}, \frac{(0.2769,0.4765,0.1872)}{i_{3}}, \\
\frac{(0.0198,0.1999,0.2888)}{i_{4}}, \frac{(0.4728,0.2981,0.1764)}{i_{5}}, \frac{(53.089,53.224,33.944)}{i_{6}}
\end{array}\right\}
$$

$$
\mathcal{A}\left(x_{6}\right)=\left\{\begin{array}{l}
\frac{(7.9447,7.3447,7.1447)}{i_{1}}, \frac{(0.8876,0.8912,0.8908)}{i_{2}}, \frac{(0.0734,0.0423,0.0981)}{i_{3}}, \\
\frac{(0.0190,0.1985,0.2896)}{i_{4}}, \frac{(0.4552,0.2732,0.1908)}{i_{5}}, \frac{(58.3892,53.2249,33.9448)}{i_{6}}
\end{array}\right\}
$$




$$
\begin{aligned}
& \mathcal{A}\left(x_{7}\right)=\left\{\begin{array}{l}
\frac{(7.9299,7.4299,7.6299)}{i_{1}}, \frac{(0.7234,0.8095,0.6987)}{i_{2}}, \frac{(0.1014,0.4982,0.1237)}{i_{3}}, \\
\frac{(0.0198,0.0194,0.0278)}{i_{4}}, \frac{(0.4552,0.2871,0.1905)}{i_{5}}, \frac{(58.389,53.224,33.944)}{i_{6}}
\end{array}\right\} \\
& \mathcal{A}\left(x_{8}\right)=\left\{\begin{array}{l}
\frac{(7.9127,7.8325,7.4325)}{i_{1}}, \frac{(0.7654,0.6981,0.9873)}{i_{2}}, \frac{(0.1413,0.4230,0.1879)}{i_{3}}, \\
\frac{(0.0188,0.0981,0.0122)}{i_{4}}, \frac{(0.4605,0.2333,0.1091)}{i_{5}}, \frac{(49.723,53.224,33.944)}{i_{6}}
\end{array}\right\} \\
& \mathcal{A}\left(x_{9}\right)=\left\{\begin{array}{l}
\frac{(7.9839,7.5325,7.3325)}{i_{1}}, \frac{(0.5981,0.6564,0.4230)}{i_{2}}, \frac{(0.3123,0.4290,0.1872)}{i_{3}}, \\
\frac{(0.5004,0.1239,0.2908)}{i_{4}}, \frac{(0.5004,0.2872,0.1891)}{i_{5}}, \frac{(52.733,53.224,33.944)}{i_{6}}
\end{array}\right\}
\end{aligned}
$$

where $\mathcal{A}\left(x_{1}\right)\left(i_{1}\right)=(6.6733,6.4325,6.7325)$ means that the entropy of S-box of $x_{1}$ is given by group 1 (resp., by group 2, by group 3 ) is 6.6733 (resp., 6.4325,6.7325); meanings of $\mathcal{A}\left(x_{s}\right)\left(i_{t}\right)$ can be explained similarly $(s=1,2,3,4,5,6,7,8,9 ; \quad t=1$, $2,3,4,5,6)$.

To find the best choice from $X$, let us first compute the 3-polar fuzzy set $\overline{\mathcal{A}} \in$ $\left([0,60]^{3}\right)^{X}$, defined by $p_{k}{ }^{\circ} \overline{\mathcal{A}}=60 \wedge \sum_{i \in I} p_{k}{ }^{\circ} \mathcal{A}(x) \quad(\forall x \in X), \quad$ where $p_{k}:[0,60]^{3} \rightarrow[0,60]$ is the $k$-the projection $(k=1,2,3)$.

$p_{1}\left(x_{1}\right)=60 \wedge(6.6733+0.2455+0.8771+0.2917+0.9334+0.0000)=9.0210 . \quad$ Similarly, (in Table 3, and Fig. 3 explained The $k$-the projection $(k=1,2,3))$.

Therefore

$$
\overline{\mathcal{A}}=\left\{\begin{array}{c}
\frac{(9.0210,7.75733,8.295)}{x_{1}}, \frac{(52.7834,60,41.4863)}{x_{2}}, \frac{(60,60,42.8005)}{x_{3}}, \frac{(52.7318,60,52.7318)}{x_{4}} \\
\frac{(60,60,42.5787)}{x_{5}}, \frac{(60,60,43.4417)}{x_{6}}, \frac{(60,60,42.6146)}{x_{7}}, \frac{(59.0217,60,42.673)}{x_{8}}, \\
\frac{(60,60,42.3666)}{x_{9}}
\end{array}\right\}
$$

Based on the weight vector $e^{\rightarrow}=(8.00,1.00,60.00)^{T}$. We compute the score $S(x)=\mathcal{A}$ $(x) e^{\rightarrow}$ for each $x \in X x \in X$. Then: (in Table 4, and Fig. 4 we define score $S(x)=\mathcal{A}(x) e^{\rightarrow}$ for each $x \in X$ )

Table 3 The $k$-the projection $(k=1,2,3)$

\begin{tabular}{llllllllll}
\hline & $x_{1}$ & $x_{2}$ & $x_{3}$ & $x_{4}$ & $x_{5}$ & $x_{6}$ & $x_{7}$ & $x_{8}$ & $x_{9}$ \\
\hline$p_{1}$ & 9.0210 & 52.7834 & 60 & 52.7318 & 60 & 60 & 60 & 59.0217 & 60 \\
$p_{2}$ & 7.5733 & 60 & 60 & 60 & 60 & 60 & 60 & 60 & 60 \\
$p_{3}$ & 8.295 & 41.4863 & 42.8005 & 52.7318 & 42.5787 & 43.4417 & 42.6146 & 42.673 & 42.3666 \\
\hline
\end{tabular}




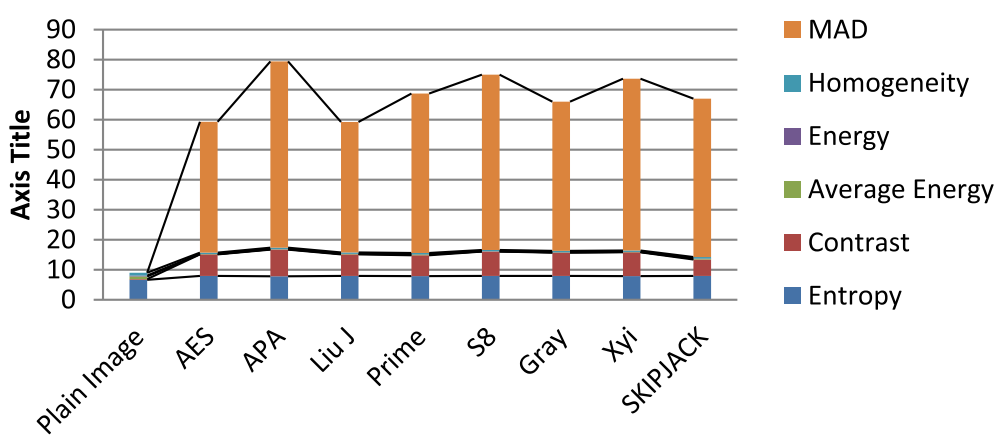

\section{Axis Title}

Fig. 3 Analyses of S-Box in image encryption applications based on fuzzy sets and 2-polar fuzzy soft set decision-making criterion, entropy, contrast, average energy, energy, homogeneity, MAD of prevailing S-box. This data explained the analyses of S-Box in image encryption applicationsbased on fuzzy sets and 2-polar fuzzy soft set decision-making criterion

As $S\left(x_{4}\right)=(52.7318,60,52.7318) e^{\rightarrow}=52.7318 \times 8.00+60 \times 1.00+52.7318 \times 60.00=3645.7$

As S-box $x_{4}$ (Lui J ) have the highest value, the best choice by experts should be Sbox $x_{4}$ (Lui J ) is the suitability of S-box to image encryption based on $m$-polar fuzzy soft set. Now, we will construct an algorithm for a decision-making problem as indicated below.

Algorithm 1: Using m-polar Fuzzy soft set.

Step 1. State $\mathcal{A} \in\left[\left([0,60]^{3}\right)^{I}\right]^{X}$.

Step 2. Compute $p_{k}^{\circ}{ }^{\circ} \overline{\mathcal{A}}=60 \wedge \sum_{i \in I} p_{k}^{\circ} \mathcal{A}(x) \quad(\forall x \in X)$, where $p_{k}:[0,60]^{3} \rightarrow[0.60]$ is the $k$-the projection $(k=1,2,3)$.

Step 3. Compute $\overline{\mathcal{A}} \in\left([0,60]^{3}\right)^{X}$.

Step 4. Put a suitable weigh vector $e^{\rightarrow}=(8.00,1.00,60.00)^{T}$. And compute the score $S(x)=$ $\mathcal{A}(x) e^{\rightarrow}$ for each $x \in X$.

Step 5. The maximal value of $S(\tilde{x})$ state the optimal alternative for suitability of S-box to image encryption Based on m-polar Fuzzy soft set.

Table 4 The score $S(x)=\mathcal{A}(x) e^{\rightarrow}$ for each $x \in X x \in X$

\begin{tabular}{|c|c|c|c|c|c|}
\hline$S\left(x_{1}\right)$ & $=$ & $(9.0210,7.75733,8.295) e^{\rightarrow}$ & $=$ & $9.0210 \times 8.00+7.75733 \times 1.00+8.295 \times 60.00$ & $=577.62$ \\
\hline$S\left(x_{2}\right)$ & $=$ & $(52.7834,60,41.4863) e^{\rightarrow}$ & $=$ & $52.7834 \times 8.00+60 \times 1.00+41.4863 \times 60.00$ & $=2971.4$ \\
\hline$S\left(x_{3}\right)$ & $=$ & $(60,60,42.8005) e^{\rightarrow}$ & $=$ & $60 \times 8.00+60 \times 1.00+42.8005 \times 60.00$ & $=3108.0$ \\
\hline$S\left(x_{4}\right)$ & $=$ & $(52.7318,60,52.7318) e^{\rightarrow}$ & $=$ & $52.7318 \times 8.00+60 \times 1.00+52.7318 \times 60.00$ & $=3645.7$ \\
\hline$S\left(x_{5}\right)$ & $=$ & $(60,60,42.5787) e^{\rightarrow}$ & $=$ & $60 \times 8.00+60 \times 1.00+42.5787 \times 60.00$ & $=3094.7$ \\
\hline$S\left(x_{6}\right)$ & $=$ & $(60,60,43.4417) e^{\rightarrow}$ & $=$ & $60 \times 8.00+60 \times 1.00+43.4417 \times 60.00$ & $=3146.5$ \\
\hline$S\left(x_{7}\right)$ & $=$ & $(60,60,42.6146) e^{\rightarrow}$ & $=$ & $60 \times 8.00+60 \times 1.00+42.6146 \times 60.00$ & $=3096.8$ \\
\hline$S\left(x_{8}\right)$ & $=$ & $(59.0217,60,42.673) e^{\rightarrow}$ & $=$ & $59.0217 \times 8.00+60 \times 1.00+42.673 \times 60.00$ & $=3092.5$ \\
\hline$S\left(x_{9}\right)$ & $=$ & $(60,60,42.3666) e^{\rightarrow}$ & $=$ & $60 \times 8.00+60 \times 1.00+42.3666 \times 60.00$ & $=3081.9$ \\
\hline
\end{tabular}




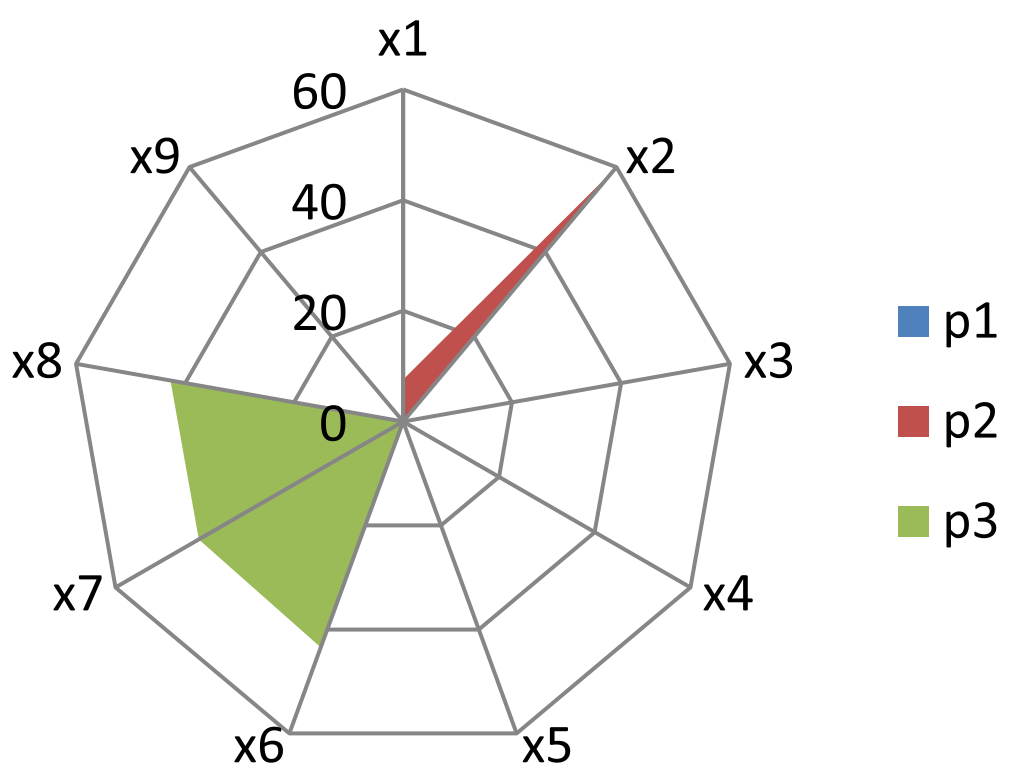

Fig. 4 The $k$-the projection $(k=1,2,3)$. This figure explained and compute the 3-polar fuzzy set $\overline{\mathcal{A}} \in\left([0,60]^{3}\right)^{X}$, defined by $p_{k}{ }^{\circ} \overline{\mathcal{A}}=60 \wedge \sum_{i \in 1} p_{k}{ }^{\circ} \mathcal{A}(x) \quad(\forall x \in X)$, where $p_{k}:[0,60]^{3} \rightarrow[0,60]$ is the $k$-the projection $(k=1,2,3)$

\section{Suitability of S-boxes to image encryption based on 2-polar fuzzy soft set}

In this part, we study analyses S-boxes (APA, Liu J, Prime, S8). There are form the set $X=\left\{x_{1}, x_{2}, x_{3}, x_{4}\right\}$ of the alternatives where $x_{i}(i=1,2,3,4)$ are S-boxes and the alternatives $x_{i}$. To evaluate the S-boxes, we take the important alternative parameters $I=\left\{i_{1}, i_{2}, i_{3}\right\}$ where $i_{1}$ stands for contrast, $i_{2}$ stands for energy, $i_{3}$ stands for homogeneity. These parameters are important with degree $(0.99,0.89$, and 0.92$)$. Considering their own needs, the data for the optimal alternative for the suitability of S-box to image encryption based on 2-polar fuzzy soft set $\mathcal{A} \in\left[\left([0,1]^{2}\right)^{X} \times\left([0,1]^{2}\right)^{X}\right]^{I}=\left[\left([0,1]^{4}\right)^{X^{I}}\right]^{I}$ $=\left[\left([0,1]^{4}\right)^{I}\right]^{X}=\left([0,1]^{4}\right)^{X \times I}$ defined by two managers to state the measure of the parameters give us the following data

$$
\begin{aligned}
& \mathcal{A}\left(x_{1}\right)=\left\{\frac{\langle(0.66,0.55),(0.88 .0 .77)\rangle}{i_{1}}, \frac{\langle(0.76,0.85),(0.786 .0 .67)\rangle}{i_{2}}, \frac{\langle(0.96,0.85),(0.68 .0 .87)\rangle}{i_{3}}\right\} \\
& \mathcal{A}\left(x_{2}\right)=\left\{\frac{\langle(0.69,0.75),(0.85 .0 .47)\rangle}{i_{1}}, \frac{\langle(0.96,0.55),(0.80 .0 .70)\rangle}{i_{2}}, \frac{\langle(0.60,0.50),(0.60 .0 .71)\rangle}{i_{3}}\right\} \\
& \mathcal{A}\left(x_{3}\right)=\left\{\frac{\langle(0.46,0.85),(0.76 .0 .87)\rangle}{i_{1}}, \frac{\langle(0.76,0.75),(0.65 .0 .67)\rangle}{i_{2}}, \frac{\langle(0.56,0.85),(0.78 .0 .87)\rangle}{i_{3}}\right\}
\end{aligned}
$$




$$
\mathcal{A}\left(x_{4}\right)=\left\{\frac{\langle(0.76,0.65),(0.86 .0 .87)\rangle}{i_{1}}, \frac{\langle(0.86,0.59),(0.87 .0 .67)\rangle}{i_{2}}, \frac{\langle(0.86,0.75),(0.83 .0 .79)\rangle}{i_{3}}\right\}
$$

Where,

$\mathcal{A}\left(x_{1}\right)\left(i_{1}\right)=\langle(0.66,0.55),(0.88 .0 .77)\rangle$. Means that of the encryption $x_{1}$ of the parameter $i_{1}$ (contrast) in the aspects of increase takes the values 0.66 or decrease takes the values 0.55 and by the second measure, the increase takes the values 0.88 or decrease takes the values 0.77 respectively; the meaning of $\mathcal{A}\left(x_{s}\right)\left(i_{t}\right)$ can be explained similarly $(s=1,2,3.4 ; t=1,2,3)$.

To find the best choice from $X$, let us first compute the 2-polar fuzzy set $\overline{\mathcal{A}} \in$ $\left([0,1]^{2}\right)^{X \times I}$ defined by

$$
\left.\overline{\mathcal{A}}(x, i)=1 \wedge \sum_{k=1}^{2}\left(p_{k}{ }^{\circ} p_{1}{ }^{\circ} \mathcal{A}(x, i) \times p_{k}{ }^{\circ} p_{2}{ }^{\circ} \mathcal{A}(x, i)\right) \quad \forall i \in I, \forall x \in X\right)
$$

where $p_{k}:[0,1]^{2} \rightarrow[0,1]$ is the $k$-the projection $(k=1,2)$;

$$
\begin{aligned}
\overline{\mathcal{A}}\left(x_{1}\right)\left(i_{1}\right) & =(0.66 \times 0.88)+(0.55 \times 0.77)=1 \wedge 1.0043=1 \text {; Similarly } \\
\overline{\mathcal{A}}\left(x_{1}\right) & =\left\{\frac{1}{i_{1}}, \frac{0.9697312}{i_{2}}, \frac{1}{i_{3}}\right\}, \overline{\mathcal{A}}\left(x_{2}\right)=\left\{\frac{0.628155}{i_{1}}, \frac{0.9226}{i_{2}}, \frac{0.6106}{i_{3}}\right\}, \overline{\mathcal{A}}\left(x_{3}\right) \\
& =\left\{\frac{1}{i_{1}}, \frac{0.83348}{i_{2}}, \frac{1}{i_{3}}\right\}, \overline{\mathcal{A}}\left(x_{4}\right)=\left\{\frac{1}{i_{1}}, \frac{0.896594}{i_{2}}, \frac{1}{i_{3}}\right\}
\end{aligned}
$$

Now, we compute $m_{i}=\sum_{k=1}^{4}\left(x_{k}\right)(i), \quad x \in X, \quad(i=1,2,3)$ and compute $r_{i}=\sum_{i}^{3} m_{i}-$ $m_{j}(j=1,2,3,4)$. (in Table 5 , and Fig. 5 we calculated $\left.r_{i}=\sum_{i}^{3}\left(m_{i}-m_{j}\right)(j=1,2,3,4)\right)$

$$
\begin{aligned}
& r_{i}=\sum_{i}^{3}\left(m_{i}-m_{j}\right) \quad(j=1,2,3,4) \text {; then } \\
& r_{1}=\left(m_{1}-m_{1}\right)+\left(\mathrm{m}_{1}-m_{2}\right)+\left(m_{1}-m_{3}\right)+\left(m_{1}-m_{4}\right)=(2.9697312-
\end{aligned}
$$

$2.9697312)+(2.9697312-1.55081606)+(2.9697312-2.83343)+(2.9697312-2.896594)$

$=1.62835354$, Similarly,

$r_{2}=-4.04730702, r_{3}=1.08314874, r_{4}=1.33580474$. Since the score $S(x)=\max r_{i}$. Then, the maximum score is $r_{1}=1.62835354$ and the optimal alternative for the suitability of S-box to image encryption based on 2-polar fuzzy soft set is to select $x_{1}$ (APA encryption ).

Table $5 r_{i}=\sum_{i}^{3}\left(m_{i}-m_{j}\right)(j=1,2,3,4)$

\begin{tabular}{lllll}
\hline$x$ & $i_{1}$ & $i_{2}$ & $i_{3}$ & $m_{i}$ \\
\hline$x_{1}$ & 1 & 0.9697312 & 1 & 2.9697312 \\
$x_{2}$ & 0.628155 & 0.9226 & 0.6106 & 1.55081606 \\
$x_{3}$ & 1 & 0.83343 & 1 & 2.83343 \\
$x_{4}$ & 1 & 0.896594 & 1 & 2.896594 \\
\hline
\end{tabular}




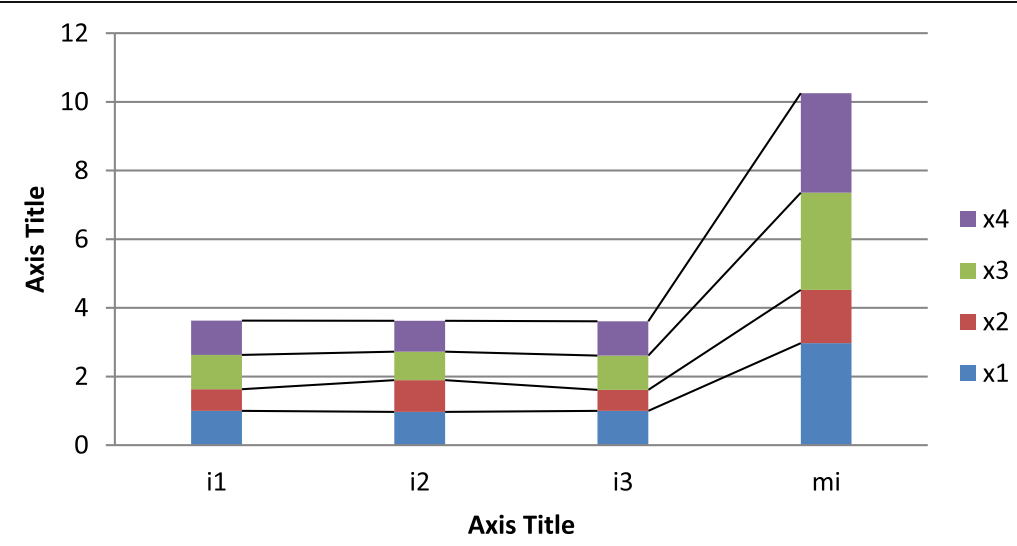

Fig. $5 r_{i}=\sum_{i}^{3}\left(m_{i}-m_{j}\right) \quad(j=1,2,3,4)$. Explained the calculation of the maximal value of score $S(x)=\max _{i} r_{i}$ to state the optimal alternative for the suitability of S-box to image encryption based on 2-polar fuzzy soft set

Motivated from the above problem, we give the following algorithm for decisionmaking problem (and the like):

Algorithm 2: Using 2-polar Fuzzy soft set.

Step 1. State $\mathcal{A} \in\left[\left([0,60]^{3}\right)^{I}\right]^{X}$

Step 1. State $\mathcal{A} \in\left[\left([0,1]^{2}\right)^{X} \times\left([0,1]^{2}\right)^{X}\right]^{I}=\left[\left([0,1]^{4}\right)^{X}\right]^{I}=\left[\left([0,1]^{4}\right)^{I}\right]^{X}=\left([0,1]^{4}\right)^{X \times I}$

Step 2. Compute the 2-polar fuzzy set $\overline{\mathcal{A}} \in\left([0,1]^{2}\right)^{X \times I}$ defined by

$$
\left.\overline{\mathcal{A}}(x, i)=1 \wedge \sum_{k=1}^{2}\left(p_{k}{ }^{\circ} p_{1}{ }^{\circ} \mathcal{A}(x, i) \times p_{k}{ }^{\circ} p_{2}{ }^{\circ} \mathcal{A}(x, i)\right) \quad \forall i \in I, \forall x \in X\right)
$$

Where $p_{k}:[0,1]^{2} \rightarrow[0,1]$ is the $k$-the projection $(k=1,2)$;

Step 3. compute $m_{i}=\sum_{k=1}^{4}\left(x_{k}\right)(i), x \in X, \quad(i=1,2,3)$ and compute $r_{i}=\sum_{i}^{3} m_{i}-m_{j} \quad(j=$ $1,2,3,4)$.

Step 4. The maximal value of score $S(x)=\max _{i} r_{i}$ to state the optimal alternative for suitability of S-box to image encryption Based on 2-polar Fuzzy soft set.

Suitability of S-boxes to image encryption based on two operations ( $\wedge$ and $\vee$ ) polar fuzzy soft sets

In this section, we study the problem by using two operations $(\wedge$ and $\vee$ ) polar fuzzy soft sets. So, we give S-boxes (Residue, Gray, AES, SKIPJACK, and Xyi). There are form the set $X=\left\{x_{1}, x_{2}, x_{3}, x_{4}, x_{5}\right\}$ of alternatives where $x_{i}\{i=1,2,3,4,5)$ are S-boxes and the alternatives $x_{i}$. To evaluate the $S$-boxes, we take the important alternative parameters $I=\left\{i_{1}, i_{2}, i_{3}\right\}$ where $i_{1}$ stands for entropy, $i_{2}$ stands for homogeneity, $i_{3}$ stands for MAD. These parameters are important with degree (8.00, 1.0, and 60.0). Considering their own needs, the data for the optimal alternative for the suitability of S-box to image encryption based on 2-polar fuzzy soft set $\mathcal{A} \in\left([0,60]^{2 \times 3}\right)^{I \times X}$ defined by: 


$$
\left.\begin{array}{c}
\mathcal{A}\left(i_{1}\right)=\left\{\begin{array}{l}
\frac{\langle(6.6,6.5),(7.6,5.7),(7.6,5.8)\rangle}{x_{1}}, \frac{\langle(6.6,6.5),(7.6,5.7),(7.6,5.8)\rangle}{x_{2}} \\
, \frac{\langle(6.6,6.5),(7.6,5.7),(7.6,5.8)\rangle}{x_{3}}, \\
\mathcal{A}\left(i_{2}\right)=\left\{\begin{array}{c}
\frac{\langle(0.6,6.5),(7.6,5.7),(7.6,5.8)\rangle}{x_{4}}, \frac{\langle(6.6,6.5),(7.6,5.7),(7.6,5.8)\rangle}{x_{5}}
\end{array}\right\} \\
\frac{\langle(0.9,0.2),(0.6,0.5),(0.8,0.2)\rangle}{x_{4}}, \frac{\langle(0.7,0.1),(0.5,0.5),(0.9,0.4)\rangle}{x_{1}}, \frac{\langle(0.5),(0.6,0.4)\rangle}{x_{1}}, \frac{\langle(0.3,0.9),(0.7,0.2),(0.6,0.4)\rangle}{x_{2}}
\end{array}\right\} \\
\mathcal{A}\left(i_{3}\right)=\left\{\begin{array}{l}
\frac{\langle(0.8),(0.9,0.1),(0.7,0.8)\rangle}{x_{2}}, \\
\frac{\langle(50.6,50.5,55.1),(45.6,42.5,45.0),(54.6,29.5,45.1)\rangle}{x_{1}}, \frac{(40.6,58.5,44.1),(60.0,50.5,25.1),(34.6,30.5,50.1)}{x_{2}} \\
\frac{\langle(30.6,50.5,45.1),(35.6,40.5,41.1),(40.6,30.5,40.1)\rangle}{x_{4}}, \frac{\langle(40.6,50.5,52.1),(35.6,42.5,45.1),(44.6,39.5,45.1)\rangle}{x_{5}}
\end{array}\right.
\end{array}\right\}
$$

where $\mathcal{A}\left(x_{1}\right)\left(i_{1}\right)=\{(6.6,6.5),(7.6,5.7),(7.6,5.8)\}$ means that of the encryption $x_{1}$ (Residue ) of the parameter $i_{1}$ (entropy) increase and decrease of growth given by the first measure is the increase takes the value 6.6 and decrease takes the value 6.5 , by the second measure, the increase takes the value 7.6 and decrease takes the value 5.7, and by the third measure, the increase takes the value 7.6 and decrease takes the value 5.7 and decrease takes the value7.6 and decrease takes the value 5.8. Respectively; the meaning of $\mathcal{A}\left(x_{s}\right)\left(i_{t}\right)$ can be explained similarly $(s=1,2$, $3.4,5 ; t=1,2,3)$. Similarly,

A subset $\mathcal{B}=\left\{B_{i}\right\}_{i}: \mathrm{I} \rightarrow\left([0,60]^{2 \times 3}\right)^{X}$ is called also 3-polar fuzzy soft set on $X$, define by $\mathcal{B}(\mathrm{i})=B_{i} \forall i \in I$, the data for optimal alternative for the suitability of S-box to image encryption based on 3-polar fuzzy soft set given by another three measures $\mathcal{B} \in$ $\left[\left([0,60]^{2 \times 3}\right)^{X}\right]^{I}=\left([0,60]^{2 \times 3}\right)^{X \times I}$ defined by

$$
\begin{gathered}
\mathcal{B}\left(i_{1}\right)=\left\{\begin{array}{c}
\frac{\langle(5.6,5.5),(6.6,4.7),(6.6,5.2)\rangle}{x_{1}}, \frac{\langle(5.6,5.5),(6.6,4.7),(6.6,4.2)\rangle}{x_{2}} \\
, \frac{\langle(4.6,6.5),(5.6,5.2),(6.6,4.8)\rangle}{x_{3}}, \\
, \frac{\langle(5.6,4.5),(4.6,4.7),(5.6,2.8)\rangle}{x_{4}}, \frac{\langle(5.1,2.5),(4.6,3.7),(5.6,3.2)\rangle}{x_{5}}
\end{array}\right\} \\
\mathcal{B}\left(i_{2}\right)=\left\{\begin{array}{l}
\frac{\langle(0.4,0.6),(0.7,0.5),(0.3,0.4)\rangle}{x_{1}}, \frac{\langle(0.3,0.2),(0.5,0.2),(0.3,0.4)\rangle}{x_{2}} \\
, \frac{\langle(0.5,0.1),(0.6,0.1),(0.4,0.2)\rangle}{x_{3}}, \\
\frac{\langle(0.5,0.2),(0.3,0.5),(0.3,0.2)\rangle}{x_{4}}, \frac{\langle(0.4,0.1),(0.1,0.5),(0.3,0.4)\rangle}{x_{5}}
\end{array}\right\}
\end{gathered}
$$


$\mathcal{B}\left(i_{3}\right)=\left\{\begin{array}{c}\frac{\langle(40.6,40.5,45.1),(40.6,40.5,41.0),(34.6,30.5,35.1)\rangle}{x_{1}}, \frac{(40.6,50.5,40.1),(60.0,50.5,25.1),(34.6,30.5,50.1)}{x_{2}} \\ , \frac{\langle(46.6,50.5,50.1),(45.6,40.5,45.1),(44.6,40.5,25.1)\rangle}{x_{3}}, \\ \frac{\langle(40.6,40.5,40.1),(30.6,40.5,40.1),(40.6,30.5,40.1)\rangle}{x_{4}}, \frac{\langle(45.6,30.5,42.1),(40.6,40.5,50.1),(40.6,59.5,45.1)\rangle}{x_{5}}\end{array}\right\}$

Now, we need to find the best choice from $X$ based on $\mathbb{C}=\mathcal{A} \wedge \mathcal{B}$. So, compute $\mathbb{C}$.

$$
\mathbb{C}\left(i_{1}, i_{1}\right)=\left\{\begin{array}{c}
\frac{\langle(5.6,5.5),(6.6,4.7),(6.6,5.2)\rangle}{x_{1}}, \frac{\langle(5.6,5.5),(6.6,4.7),(6.6,4.2)\rangle}{x_{2}} \\
\frac{\langle(4.6,6.5),(5.6,5.2),(6.6,4.8)\rangle^{2}}{x_{3}}, \\
\frac{\langle(5.6,4.5),(4.6,4.7),(5.6,2.8)\rangle}{x_{4}}, \frac{\langle(5.1,2.5),(4.6,3.7),(5.6,3.2)\rangle}{x_{5}}
\end{array}\right\}
$$

$C\left(i_{1}, i_{2}\right)==\left\{\begin{array}{c}\frac{\langle(0.4,0.6),(0.7,0.5),(0.3,0.4)\rangle}{x_{1}}, \frac{\langle(0.3,0.2),(0.5,0.2),(0.3,0.4)\rangle}{x_{2}} \\ \frac{\langle(0.5,0.1),(0.6,0.1),(0.4,0.2)\rangle^{2}}{x_{3}}, \\ \frac{\langle(0.5,0.2),(0.3,0.5),(0.3,0.2)\rangle}{x_{4}}, \frac{\langle(0.4,0.1),(0.1,0.5),(0.3,0.4)\rangle}{x_{5}}\end{array}\right\}$

$$
\mathbb{C}\left(i_{1}, i_{3}\right)=\left\{\begin{array}{c}
\frac{\langle(6.6,6.5),(7.6,5.7),(7.6,5.8)\rangle}{x_{1}}, \frac{\langle(6.6,6.5),(7.6,5.7),(7.6,5.8)\rangle}{x_{2}} \\
\frac{\langle(6.6,6.5),(7.6,5.7),(7.6,5.8)\rangle^{2}}{x_{3}}, \\
\frac{\langle(6.6,6.5),(7.6,5.7),(7.6,5.8)\rangle}{x_{4}}, \frac{\langle(6.6,6.5),(7.6,5.7),(7.6,5.8)\rangle}{x_{5}}
\end{array}\right\}
$$

$$
\mathbb{C}\left(i_{2}, i_{1}\right)=\left\{\begin{array}{c}
\frac{\langle(0.6,0.5),(0.8,0.5),(0.6,0.4)\rangle}{x_{1}}, \frac{\langle(0.3,0.9),(0.7,0.2),(0.6,0.4)\rangle}{x_{2}} \\
\frac{\langle(0.5,0.8),(0.9,0.1),(0.7,0.8)\rangle}{x_{3}}, \\
\frac{\langle(0.9,0.2),(0.6,0.5),(0.8,0.2)\rangle}{x_{4}}, \frac{\langle(0.7,0.1),(0.5,0.5),(0.9,0.4)\rangle}{x_{5}}
\end{array}\right\}
$$

$$
\mathbb{C}\left(i_{2}, i_{2}\right)=\left\{\begin{array}{c}
\frac{\langle(0.4,0.5),(0.7,0.5),(0.3,0.4)\rangle}{x_{1}}, \frac{\langle(0.3,0.2),(0.5,0.2),(0.3,0.4)\rangle}{x_{2}} \\
\frac{\langle(0.5,0.1),(0.6,0.1),(0.4,0.2)\rangle^{2}}{x_{3}}, \\
\frac{\langle(0.5,0.2),(0.3,0.5),(0.3,0.2)\rangle}{x_{4}}, \frac{\langle(0.4,0.1),(0.1,0.5),(0.3,0.4)\rangle}{x_{5}}
\end{array}\right\}
$$




$$
\begin{aligned}
& \mathbb{C}\left(i_{2}, i_{3}\right)=\left\{\begin{array}{c}
\frac{\langle(0.6,0.5),(0.8,0.5),(0.6,0.4)\rangle}{x_{1}}, \frac{\langle(0.3,0.9),(0.7,0.2),(0.6,0.4)\rangle}{x_{2}} \\
\frac{\langle(0.5,0.8),(0.9,0.1),(0.7,0.8)\rangle}{x_{3}}, \\
\frac{\langle(0.9,0.2),(0.6,0.5),(0.8,0.2)\rangle}{x_{4}}, \frac{\langle(0.7,0.1),(0.5,0.5),(0.9,0.4)\rangle}{x_{5}}
\end{array}\right\} \\
& \mathbb{C}\left(i_{3}, i_{1}\right)=\left\{\begin{array}{c}
\frac{\langle(5.6,5.5),(6.6,4.7),(6.6,5.2)\rangle}{x_{1}}, \frac{\langle(5.6,5.5),(6.6,4.7),(6.6,4.2)\rangle}{x_{2}} \\
\frac{\langle(4.6,6.5),(5.6,5.2),(6.6,4.8)\rangle^{2}}{x_{3}}, \\
\frac{\langle(5.6,4.5),(4.6,4.7),(5.6,2.8)\rangle}{x_{4}}, \frac{\langle(5.1,2.5),(4.6,3.7),(5.6,3.2)\rangle}{x_{5}}
\end{array}\right\} \\
& \mathbb{C}\left(i_{3}, i_{2}\right)=\left\{\begin{array}{c}
\frac{\langle(0.4,0.6),(0.7,0.5),(0.3,0.4)\rangle}{x_{1}}, \frac{\langle(0.3,0.2),(0.5,0.2),(0.3,0.4)\rangle}{x_{2}} \\
\frac{\langle(0.5,0.1),(0.6,0.1),(0.4,0.2)\rangle^{2}}{x_{3}}, \\
\frac{\langle(0.5,0.2),(0.3,0.5),(0.3,0.2)\rangle}{x_{4}}, \frac{\langle(0.4,0.1),(0.1,0.5),(0.3,0.4)\rangle}{x_{5}}
\end{array}\right\} \\
& C\left(i_{3}, i_{3}\right)=\left\{\begin{array}{c}
\frac{\langle(40.6,40.5,45.1),(40.6,40.5,41.0),(34.6,30.5,35.1)\rangle}{x_{1}}, \frac{(40.6,50.5,40.1),(60.0,50.5,25.1),(34.6,30.5,50.1)}{x_{2}} \\
\frac{\langle(46.6,50.5,50.1),(45.6,40.5,45.1),(44.6,39.5,25.1)\rangle}{x_{3}}, \\
\frac{\langle(30.6,40.5,40.1),(30.6,40.5,40.1),(40.6,30.5,40.1)\rangle}{x_{4}}, \frac{\langle(40.6,30.5,42.1),(35.6,40.5,45.1),(40.6,39.5,45.1)\rangle}{x_{5}}
\end{array}\right\}
\end{aligned}
$$

Secondly, compute the 3-polar fuzzy soft set $\hat{\mathbb{C}} \in\left([0,60]^{2 \times 3}\right)^{X}$, defined by

$$
\left.\hat{\mathbb{C}}(x)(i, j)=60 \wedge \sum_{k=1}^{3}\left(p_{k}{ }^{\circ} p_{1}{ }^{\circ} \mathbb{C}(x)(i, j) \times p_{k}{ }^{\circ} p_{2}{ }^{\circ} \mathbb{C}(x)(i, j)\right) \quad \forall(i, j) \in I^{2}, \forall x \in X\right)
$$

where $p_{k}:[0,60]^{2} \rightarrow[0,60]$ is the $k$-the projection $(k=1,2,3)$;

$\hat{\mathbb{C}}\left(x_{1}\right)\left(i_{1}, j_{1}\right)=(60) \wedge[(5.6 \times 6.6 \times 6.6)+(5.5 \times 4.7 \times 5.2)]=(60) \wedge(6096.21584)=60 ;$

Similarly; (in Table 6, and Fig. 6 we define the values of $\hat{\mathbb{C}}(x)(i, j) \in\left([0,60]^{2 \times 3}\right)^{X}$ ).

Therefore,

$$
\begin{aligned}
& \hat{\mathbb{C}}\left(x_{1}\right)=\left\{\frac{60}{\left(i_{1}, j_{1}\right)}, \frac{0.1368}{\left(i_{1}, j_{2}\right)}, \frac{60}{\left(i_{1}, j_{3}\right)}, \frac{0.1576}{\left(i_{2}, j_{1}\right)}, \frac{0.1168}{\left(i_{2}, j_{2}\right)}, \frac{0.1576}{\left(\left(i_{2}, j_{3}\right)\right)}, \frac{60}{\left(i_{3}, j_{1}\right)}, \frac{0.1368}{\left.i_{3}, j_{2}\right)}, \frac{60}{\left(i_{3}, j_{3}\right)}\right\} \\
& \hat{\mathbb{C}}\left(x_{2}\right)=\left\{\frac{60}{\left(i_{1}, j_{1}\right)}, \frac{0.0196}{\left(i_{1}, j_{2}\right)}, \frac{60}{\left(i_{1}, j_{3}\right)}, \frac{0.03408}{\left(i_{2}, j_{1}\right)}, \frac{0.0196}{\left(i_{2}, j_{2}\right)}, \frac{0.11736}{\left(\left(i_{2}, j_{3}\right)\right)}, \frac{60}{\left(i_{3}, j_{1}\right)}, \frac{0.0196}{\left.i_{3}, j_{2}\right)}, \frac{60}{\left(i_{3}, j_{3}\right)}\right\} \\
& \hat{\mathbb{C}}\left(x_{3}\right)=\left\{\frac{60}{\left(i_{1}, j_{1}\right)}, \frac{60}{\left(i_{1}, j_{2}\right)}, \frac{60}{\left(i_{1}, j_{3}\right)}, \frac{0.0892}{\left(i_{2}, j_{1}\right)}, \frac{0.0032}{\left(i_{2}, j_{2}\right)}, \frac{0.0892}{\left(\left(i_{2}, j_{3}\right)\right)}, \frac{60}{\left(i_{3}, j_{1}\right)}, \frac{0.0044}{\left.i_{3}, j_{2}\right)}, \frac{60}{\left(i_{3}, j_{3}\right)}\right\} \\
& \hat{\mathbb{C}}\left(x_{4}\right)=\left\{\frac{60}{\left(i_{1}, j_{1}\right)}, \frac{60}{\left(i_{1}, j_{2}\right)}, \frac{60}{\left(i_{1}, j_{3}\right)}, \frac{0.0632}{\left(i_{2}, j_{1}\right)}, \frac{0.0245}{\left(i_{2}, j_{2}\right)}, \frac{0.0632}{\left(\left(i_{2}, j_{3}\right)\right)}, \frac{60}{\left(i_{3}, j_{1}\right)}, \frac{0.0245}{\left.i_{3}, j_{2}\right)}, \frac{60}{\left(i_{3}, j_{3}\right)}\right\}
\end{aligned}
$$


$\underline{\text { Table } 6 \hat{\mathbb{C}}(x)(i, j) \in\left([0,60]^{2 \times 3}\right)^{x}}$

\begin{tabular}{lccccc}
\hline$\hat{\mathbb{C}}$ & $\hat{\mathbb{C}}\left(x_{1}\right)$ & $\hat{\mathbb{C}}\left(x_{2}\right)$ & $\hat{\mathbb{C}}\left(x_{3}\right)$ & $\hat{\mathbb{C}}\left(x_{4}\right)$ & $\hat{\mathbb{C}}\left(x_{5}\right)$ \\
\hline$\left(i_{1}, j_{1}\right)$ & 60 & 60 & 60 & 60 & 60 \\
$\left(i_{1}, j_{2}\right)$ & 0.1368 & 0.0196 & 60 & 60 & 60 \\
$\left(i_{1}, j_{3}\right)$ & 60 & 60 & 60 & 60 & 60 \\
$\left(i_{2}, j_{1}\right)$ & 0.1576 & 0.03408 & 0.0892 & 0.0632 & 0.083 \\
$\left(i_{2}, j_{2}\right)$ & 0.1168 & 0.0196 & 0.0032 & 0.0245 & 0.0022 \\
$\left(i_{2}, j_{3}\right)$ & 0.1576 & 0.11736 & 0.0892 & 0.0632 & 0.083 \\
$\left(i_{3}, j_{1}\right)$ & 60 & 60 & 60 & 60 & 60 \\
$\left(i_{3}, j_{2}\right)$ & 0.1368 & 0.0196 & 0.0044 & 0.0245 & 0.0224 \\
$\left(i_{3}, j_{3}\right)$ & 60 & 60 & 60 & 60 & 60 \\
\hline
\end{tabular}

$\hat{C}\left(x_{5}\right)=\left\{\frac{60}{\left(i_{1}, j_{1}\right)}, \frac{60}{\left(i_{1}, j_{2}\right)}, \frac{60}{\left(i_{1}, j_{3}\right)}, \frac{0.083}{\left(i_{2}, j_{1}\right)}, \frac{0.0022}{\left(i_{2}, j_{2}\right)}, \frac{0.083}{\left(\left(i_{2}, j_{3}\right)\right)}, \frac{60}{\left(i_{3}, j_{1}\right)}, \frac{0.0224}{\left.i_{3}, j_{2}\right)}, \frac{60}{\left(i_{3}, j_{3}\right)}\right\}$

Now, we make a decision by two ways:

(1) First way:

Define a mapping $\mathbb{C}_{M}: X \rightarrow R$,by $\mathbb{C}_{M}(x)=\sum_{(i, j) \in I^{2}} \beta(x)(i, j)$, where

$$
\beta(x)(i, j)=\left\{\begin{array}{cc}
\mathbb{C}(x)(i, j), & \mathbb{C}(x)(i, j)=\max \left\{\mathbb{C}(x)(s, t):(s, t) \in I^{2}\right\}, \\
0 & \text { 0therwise. }
\end{array}\right.
$$

From the following table; (Table 7 and Fig. 7 , we define the mapping $\mathbb{C}_{M}: X \rightarrow R$, by $\left.\mathbb{C}_{M}(x)=\sum_{(i, j) \in I^{2}} \beta(x)(i, j)\right)$

Since $\mathbb{C}_{M}\left(\left(x_{1}\right)=120.5688=\max \mathbb{C}_{M}\right.$, then the optimal alternative for the suitability of S-box to image encryption $\mathrm{X}$ based on 3-polar fuzzy soft set is $x_{1}$ (Residue encryption).

Motivated from the above problem, we give the following algorithm for decisionmaking problem:

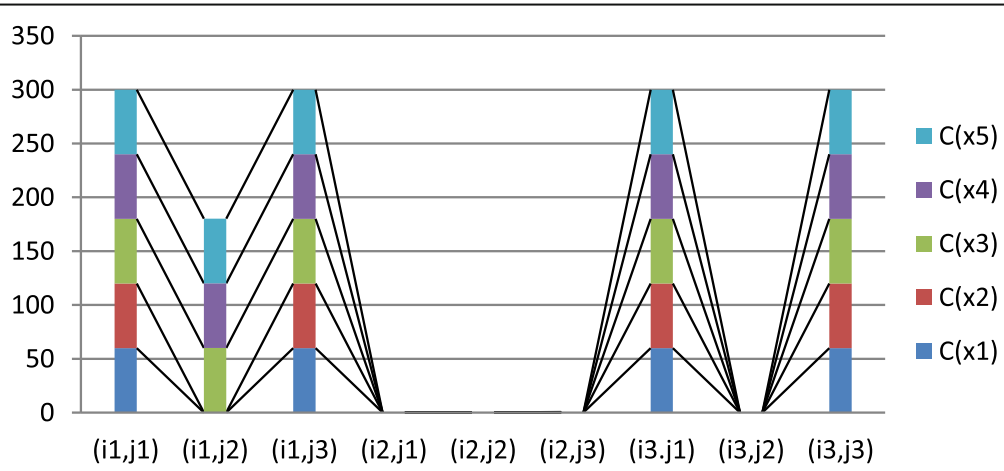

Fig. $6 \hat{\mathbb{C}}(x)(i, j) \in\left([0,60]^{2 \times 3}\right)^{x}$. This figure explained and compute the 3-polar Fuzzy soft set $\hat{\mathbb{C}} \in\left([0,60]^{2 \times 3}\right)^{x}$, defined by $\left.\hat{\mathbb{C}}(x)(i, j)=60 \wedge \sum_{k=1}^{3}\left(p_{k}{ }^{\circ} p_{1}{ }^{\circ} \mathbb{C}(x)(i, j) \times p_{k}{ }^{\circ} p_{2}{ }^{\circ} \mathbb{C}(x)(i, j)\right) \quad \forall(i, j) \in I^{2}, \forall x \in X\right)$ where $p_{k}:[0$, $60]^{2} \rightarrow[0,60]$ is the $k$-the projection $(k=1,2,3)$ 
Table 7 Mapping $\mathbb{C}_{M}: X \rightarrow R$,by $\mathbb{C}_{M}(x)=\sum_{(i, j) \in 1^{2}} \beta(x)(i, j)$

\begin{tabular}{|c|c|c|c|c|c|}
\hline$\hat{\mathbb{C}}$ & $\hat{\mathbb{C}}\left(x_{1}\right)$ & $\hat{\mathbb{C}}\left(x_{2}\right)$ & $\hat{\mathbb{C}}\left(x_{3}\right)$ & $\hat{\mathbb{C}}\left(x_{4}\right)$ & $\hat{\mathbb{C}}\left(x_{5}\right)$ \\
\hline$\left(i_{1}, j_{1}\right)$ & 60 & 60 & 60 & $\underline{60}$ & 60 \\
\hline$\left(i_{1}, j_{2}\right)$ & 0.1368 & 0.0196 & $\underline{60}$ & 60 & 60 \\
\hline$\left(i_{1}, j_{3}\right)$ & 60 & $\underline{60}$ & 60 & 60 & 60 \\
\hline$\left(i_{2}, j_{1}\right)$ & $\underline{0.1576}$ & 0.03408 & 0.0892 & 0.0632 & 0.083 \\
\hline$\left(i_{2}, j_{2}\right)$ & $\underline{0.1168}$ & 0.0196 & 0.0032 & 0.0245 & 0.0022 \\
\hline$\left(i_{2}, j_{3}\right)$ & $\underline{0.1576}$ & 0.11736 & 0.0892 & 0.0632 & 0.083 \\
\hline$\left(i_{3}, j_{1}\right)$ & $\underline{60}$ & 60 & 60 & 60 & 60 \\
\hline$\left(i_{3}, j_{2}\right)$ & $\underline{0.1368}$ & 0.0196 & 0.0044 & 0.0245 & 0.0224 \\
\hline$\left(i_{3}, j_{3}\right)$ & $\underline{60}$ & 60 & 60 & 60 & 60 \\
\hline $\mathbb{C}_{M}$ & 120.5688 & 60 & 60 & 60 & 0 \\
\hline
\end{tabular}

Algorithm 3: Using 3-polar Fuzzy soft set.

Step 1. State $\mathcal{A}, \mathcal{B} \in\left[\left([0,60]^{2}\right)^{X} \times\left([0,60]^{2}\right)^{X}\right]^{I}=\left[\left([0,60]^{4}\right)^{X}\right]^{I}=\left[\left([0,60]^{4}\right)^{I}\right]^{X}=\left([0,60]^{4}\right)^{X \times I}$

Step 2. Compute $\mathbb{C}=\mathcal{A} \wedge \mathcal{B} \in\left[\left([0,60]^{2}\right)^{X} \times\left([0,60]^{2}\right)^{X}\right]^{I}$

Step 3. Compute the 3-polar Fuzzy soft set $\widehat{\mathbb{C}} \in\left([0,60]^{2 \times 3}\right)^{X}$, defined by

$$
\left.\widehat{\mathbb{C}}(x)(i, j)=60 \wedge \sum_{k=1}^{3}\left(p_{k}{ }^{\circ} p_{1}{ }^{\circ} \mathbb{C}(x)(i, j) \times p_{k}{ }^{\circ} p_{2}{ }^{\circ} \mathbb{C}(x)(i, j)\right) \quad \forall(i, j) \in I^{2}, \forall x \in X\right)
$$

Where $p_{k}:[0,60]^{2} \rightarrow[0,60]$ is the $k$-the projection $(k=1,2,3)$;

Step 4. $\mathbb{C}_{M}: X \rightarrow R$, by $\mathbb{C}_{M}(x)=\sum_{(i, j) \in I^{2}} \beta(x)(i, j)$, where

$$
\beta(x)(i, j)=\left\{\begin{array}{cc}
\mathbb{C}(x)(i, j), & \mathbb{C}(x)(i, j)=\max \left\{\mathbb{C}(x)(s, t):(s, t) \in I^{2}\right\}, \\
0 & \text { 0therwise. }
\end{array}\right.
$$

Step 5. The maximal value of $\mathbb{C}_{M}$ to state the optimal alternative for suitability of S-box to image encryption based on 3-polar Fuzzy soft set.

(2) Second way:

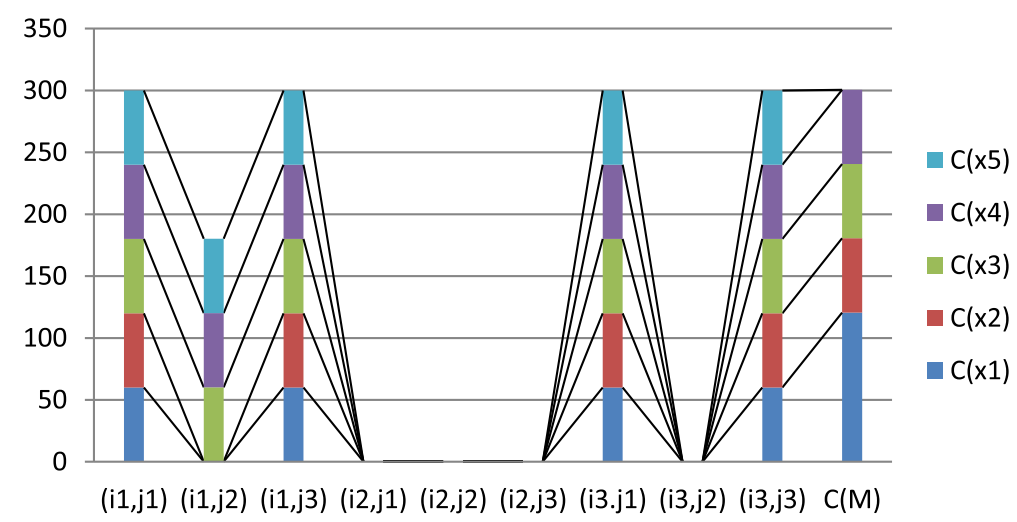

Fig. 7 Mapping $\mathbb{C}_{M}: X \rightarrow R$, by $\mathbb{C}_{M}(X)=\sum_{\left.(i, j) \in\right|^{2}} \beta(x)(i, j)$. Explained and defined a mapping $\mathbb{C}_{M}: X \rightarrow R$,by $\mathbb{C}_{M}(x)=\sum_{\left.(i, j) \in\right|^{2}} \beta(x)(i, j)$ 
Compute $m_{i}=\sum_{k=1}^{5}\left(x_{k}\right)(i, j), \quad x \in X, \quad(i, j) \in(I \times I)$ and compute $r_{i}=\sum_{j=1}^{9}\left(m_{i}-m_{j}\right)$ $(i=1,2,3,4,5)$, then, (in Table 8 and Fig. 8 we compute $r_{i}=\sum_{j=1}^{3}\left(m_{i}-m_{j}\right) \quad(i=1$, $2,3,4,5))$

$r_{1}=\left(m_{1}-m_{1}\right)+\left(\mathrm{m}_{1}-m_{2}\right)+\left(m_{1}-m_{3}\right)+\left(m_{1}-m_{4}\right)+\left(m_{1}-m_{5}\right)=(540-540)+(540$

$-360.176)+(540-540)+(540-0.69656)+(540-0.2156)=1618.87224$; similarity,

$r_{2}=359.79184, \quad r_{3}=1079.08784, r_{4}=-1437.60536, \quad r_{5}=-1440.01016$. Since $r_{1}=$

$1618.87224=\max r_{i}, \quad$ then the optimal alternative for the suitability of S-box to image encryption X based on 3-polar fuzzy soft set is $x_{1}$ (Residue encryption).

Motivated from the above problem, we give the following algorithm for decisionmaking problem:

Algorithm 4: Using 3-polar Fuzzy soft set.

Step 1. State $\mathcal{A}, \mathcal{B} \in\left[\left([0,60]^{2}\right)^{X} \times\left([0,60]^{2}\right)^{X}\right]^{I}=\left[\left([0,60]^{4}\right)^{X}\right]^{I}=\left[\left([0,60]^{4}\right)^{I}\right]^{X}=\left([0,60]^{4}\right)^{X \times I}$

Step 2. Compute $\mathbb{C}=\mathcal{A} \wedge \mathcal{B} \in\left[\left([0,60]^{2}\right)^{X} \times\left([0,60]^{2}\right)^{X}\right]^{I}$

Step 3. Compute the 3-polar Fuzzy soft set $\widehat{\mathbb{C}} \in\left([0,60]^{2 \times 3}\right)^{X}$, defined by

$$
\left.\widehat{\mathbb{C}}(x)(i, j)=60 \wedge \sum_{k=1}^{3}\left(p_{k}{ }^{\circ} p_{1}{ }^{\circ} \mathbb{C}(x)(i, j) \times p_{k}{ }^{\circ} p_{2}{ }^{\circ} \mathbb{C}(x)(i, j)\right) \quad \forall(i, j) \in I^{2}, \forall x \in X\right)
$$

Where $p_{k}:[0,60]^{2} \rightarrow[0,60]$ is the $k$-the projection $(k=1,2,3)$;

Step 4. Compute $m_{i}=\sum_{k=1}^{5}\left(x_{k}\right)(i, j), x \in X,(i, j) \in(I \times I)$ and compute $r_{i}=\sum_{j=1}^{9}\left(m_{i}-\right.$ $\left.m_{j}\right)(i=1,2,3,4,5)$,

Step 5. The maximal value of $r_{i}=\sum_{j=1}^{3}\left(m_{i}-m_{j}\right) \quad(i, j=1,2,3,4,5)$ to state the optimal alternative for suitability of S-box to image encryption based on 3-polar Fuzzy soft set.

Now, find the optimal alternative for the suitability of S-box to image encryption based on 3-polar fuzzy soft set. By using the operatorv,

First, compute $\mathbb{C}=\mathcal{A} \vee \mathcal{B}$. So, compute $\mathbb{C}$.

$$
\mathbb{C}\left(i_{1}, i_{1}\right)=\left\{\begin{array}{c}
\frac{\langle(6.6,6.5),(7.6,5.7),(7.6,5.8)\rangle}{x_{1}}, \frac{\langle(6.6,6.5),(7.6,5.7),(7.6,5.8)\rangle}{x_{2}} \\
\frac{\langle(6.6,6.5),(7.6,5.7),(7.6,5.8)\rangle}{x_{3}}, \\
, \frac{\langle(6.6,6.5),(7.6,5.7),(7.6,5.8)\rangle}{x_{4}}, \frac{\langle(6.6,6.5),(7.6,5.7),(7.6,5.8)\rangle}{x_{5}}
\end{array}\right\}
$$

\begin{tabular}{|c|c|c|c|c|c|c|c|c|c|c|}
\hline & $\left(i_{1}, j_{1}\right)$ & $\left(i_{1}, j_{2}\right)$ & $\left(i_{1}, j_{3}\right)$ & $\left(i_{2}, j_{1}\right)$ & $\left(i_{2}, j_{2}\right)$ & $\left(i_{2}, j_{3}\right)$ & $\left(i_{3}, j_{1}\right)$ & $\left(i_{3}, j_{2}\right)$ & $\left(i_{3}, j_{3}\right)$ & $m_{i}$ \\
\hline$x_{1}$ & 60 & 60 & 60 & 60 & 60 & 60 & 60 & 60 & 60 & 540 \\
\hline$x_{2}$ & 0.1368 & 0.0196 & 60 & 60 & 60 & 0.0196 & 60 & 60 & 60 & 360.176 \\
\hline$x_{3}$ & 60 & 60 & 60 & 60 & 60 & 60 & 60 & 60 & 60 & 540 \\
\hline$x_{4}$ & 0.1576 & 0.03408 & 0.0892 & 0.0632 & 0.083 & 0.03408 & 0.0892 & 0.0632 & 0.083 & 0.69656 \\
\hline$x_{5}$ & 0.1168 & 0.0196 & 0.0032 & 0.0245 & 0.0022 & 0.0196 & 0.0032 & 0.0245 & 0.0022 & 0.2156 \\
\hline
\end{tabular}

Table 8 Compute $r_{i}=\sum_{j=1}^{3}\left(m_{i}-m_{j}\right) \quad(i, j=1,2,3,4,5)$ 


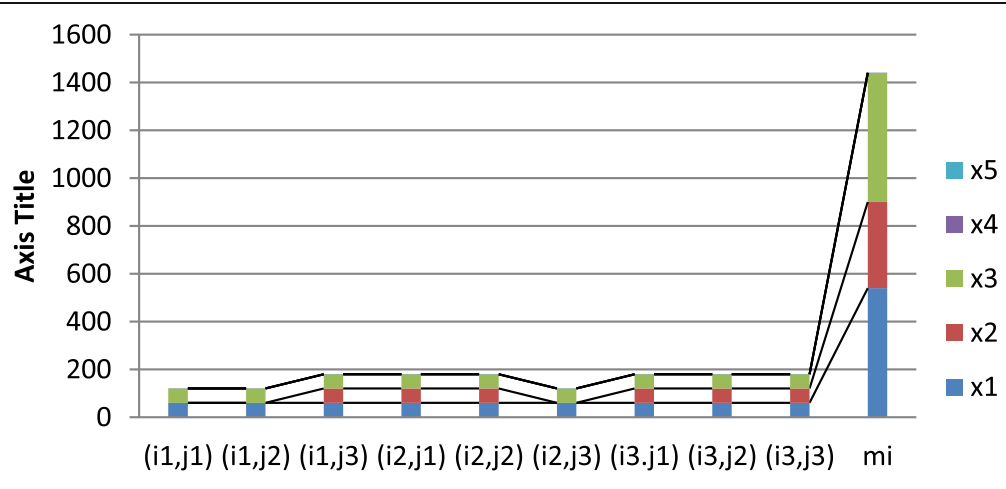

\section{Axis Title}

Fig. 8 Compute $r_{i}=\sum_{j=1}^{3}\left(m_{i}-m_{j}\right) \quad(i, j=1,2,3,4,5)$. Compute $m_{i}=\sum_{k=1}^{5}\left(x_{k}\right)(i, j), \quad x \in X, \quad(i, j) \in(I \times /$ ) and compute $r_{i}=\sum_{j=1}^{9}\left(m_{i}-m_{j}\right) \quad(i=1,2,3,4,5)$

$$
C\left(i_{1}, i_{2}\right)=\left\{\begin{array}{c}
\frac{\langle(6.6,6.5),(7.6,5.7),(7.6,5.8)\rangle}{x_{1}}, \frac{\langle(6.6,6.5),(7.6,5.7),(7.6,5.8)\rangle}{x_{2}} \\
, \frac{\langle(6.6,6.5),(7.6,5.7),(7.6,5.8)\rangle}{x_{3}}, \\
\frac{\langle(6.6,6.5),(7.6,5.7),(7.6,5.8)\rangle}{x_{4}}, \frac{\langle(6.6,6.5),(7.6,5.7),(7.6,5.8)\rangle}{x_{5}}
\end{array}\right\}
$$

$C\left(i_{1}, i_{3}\right)=\left\{\begin{array}{c}\frac{\langle(40.6,40.5,45.1),(40.6,40.5,41.0),(34.6,30.5,35.1)\rangle}{x_{1}}, \frac{(40.6,50.5,40.1),(60.0,50.5,25.1),(34.6,30.5,50.1)}{x_{2}} \\ , \frac{\langle(46.6,50.5,50.1),(45.6,40.5,45.1),(44.6,40.5,25.1)\rangle}{x_{3}}, \\ \frac{\langle(40.6,40.5,40.1),(30.6,40.5,40.1),(40.6,30.5,40.1)\rangle}{x_{4}}, \frac{\langle(45.6,30.5,42.1),(40.6,40.5,50.1),(40.6,59.5,45.1)\rangle}{x_{5}}\end{array}\right\}$

$$
\mathbb{C}\left(i_{2}, i_{1}\right)=\left\{\begin{array}{c}
\frac{\langle(5.6,5.5),(6.6,4.7),(6.6,5.2)\rangle}{x_{1}}, \frac{\langle(5.6,5.5),(6.6,4.7),(6.6,4.2)\rangle}{x_{2}} \\
\frac{\langle(4.6,6.5),(5.6,5.2),(6.6,4.8)\rangle^{2}}{x_{3}}, \\
\frac{\langle(5.6,4.5),(4.6,4.7),(5.6,2.8)\rangle}{x_{4}}, \frac{\langle(5.1,2.5),(4.6,3.7),(5.6,3.2)\rangle}{x_{5}}
\end{array}\right\}
$$

$$
\mathbb{C}\left(i_{2}, i_{2}\right)=\left\{\begin{array}{c}
\frac{\langle(0.6,0.6),(0.8,0.5),(0.6,0.4)\rangle}{x_{1}}, \frac{\langle(0.3,0.9),(0.7,0.2),(0.6,0.4)\rangle}{x_{2}} \\
\frac{\langle(0.5,0.8),(0.9,0.1),(0.7,0.8)\rangle^{-}}{x_{3}}, \\
\frac{\langle(0.9,0.2),(0.6,0.5),(0.8,0.2)\rangle}{x_{4}}, \frac{\langle(0.7,0.1),(0.5,0.5),(0.9,0.4)\rangle}{x_{5}}
\end{array}\right\}
$$

$C\left(i_{2}, i_{3}\right)=\left\{\begin{array}{c}\frac{\langle(40.6,40.5,45.1),(40.6,40.5,41.0),(34.6,30.5,35.1)\rangle}{x_{1}}, \frac{(40.6,50.5,40.1),(60.0,50.5,25.1),(34.6,30.5,50.1)}{x_{2}} \\ , \frac{\langle(46.6,50.5,50.1),(45.6,40.5,45.1),(44.6,40.5,25.1)\rangle}{x_{3}}, \\ \frac{\langle(40.6,40.5,40.1),(30.6,40.5,40.1),(40.6,30.5,40.1)\rangle}{x_{4}}, \frac{\langle(45.6,30.5,42.1),(40.6,40.5,50.1),(40.6,59.5,45.1)\rangle}{x_{5}}\end{array}\right\}$ 
$C\left(i_{3}, i_{1}\right)=\left\{\begin{array}{c}\frac{\langle(50.6,50.5,55.1),(45.6,42.5,45.0),(54.6,29.5,45.1)\rangle}{x_{1}}, \frac{(40.6,58.5,44.1),(60.0,50.5,25.1),(34.6,30.5,50.1)}{x_{2}} \\ \frac{\langle(46.6,50.5,50.1),(45.6,40.5,45.1),(44.6,39.5,45.1)\rangle}{x_{3}}, \\ \frac{\langle(30.6,50.5,45.1),(35.6,40.5,41.1),(40.6,30.5,40.1)\rangle}{x_{4}}, \frac{\langle(40.6,50.5,52.1),(35.6,42.5,45.1),(44.6,39.5,45.1)\rangle}{x_{5}}\end{array}\right\}$

$C\left(i_{3}, i_{2}\right)=\left\{\begin{array}{c}\frac{\langle(50.6,50.5,55.1),(45.6,42.5,45.0),(54.6,29.5,45.1)\rangle}{x_{1}}, \frac{(40.6,58.5,44.1),(60.0,50.5,25.1),(34.6,30.5,50.1)}{x_{2}} \\ \frac{\langle(46.6,50.5,50.1),(45.6,40.5,45.1),(44.6,39.5,45.1)\rangle}{x_{3}}, \\ \frac{\langle(30.6,50.5,45.1),(35.6,40.5,41.1),(40.6,30.5,40.1)\rangle}{x_{4}}, \frac{\langle(40.6,50.5,52.1),(35.6,42.5,45.1),(44.6,39.5,45.1)\rangle}{x_{5}}\end{array}\right\}$

$C\left(i_{3}, i_{3}\right)=\left\{\begin{array}{c}\frac{\langle(50.6,50.5,55.1),(45.6,42.5,45.0),(54.6,30.5,45.1)\rangle}{x_{1}}, \frac{(40.6,58.5,44.1),(60.0,50.5,25.1),(34.6,30.5,50.1)}{x_{2}} \\ , \frac{\langle(46.6,50.5,50.1),(45.6,40.5,45.1),(44.6,40.5,45.1)\rangle}{x_{3}}, \\ \frac{\langle(40.6,50.5,45.1),(35.6,40.5,41.1),(40.6,30.5,40.1)\rangle}{x_{4}}, \frac{\langle(40.6,50.5,52.1),(40.6,42.5,45.1),(44.6,59.5,45.1)\rangle}{x_{5}}\end{array}\right\}$

Secondly, compute the 3-polar fuzzy soft set $\hat{C} \in\left([0,60]^{2 \times 3}\right)^{X}$, defined by

$$
\left.\hat{C}(x)(i, j)=60 \wedge \sum_{k=1}^{3}\left(p_{k}{ }^{\circ} p_{1}{ }^{\circ} \mathbb{C}(x)(i, j) \times p_{k}{ }^{\circ} p_{2}{ }^{\circ} \mathbb{C}(x)(i, j)\right) \quad \forall(i, j) \in I^{2}, \forall x \in X\right)
$$

Where $p_{k}:[0,60]^{2} \rightarrow[0,60]$ is the $k$-the projection $(k=1,2,3)$;

$$
\hat{C}\left(x_{1}\right)\left(i_{1}, j_{1}\right)=(60) \wedge[(6.6 \times 7.6 \times 7.6)+(6.6 \times 7.6 \times 7.6)]=(60) \wedge(762.432)=60 ;
$$

Similarly, in Table 9, and Fig. 9 we compute $\hat{\mathbb{C}}(x)(i, j))$

Therefore,

$$
\begin{aligned}
& \hat{\mathbb{C}}\left(x_{1}\right)=\left\{\frac{60}{\left(i_{1}, j_{1}\right)}, \frac{60}{\left(i_{1}, j_{2}\right)}, \frac{60}{\left(i_{1}, j_{3}\right)}, \frac{60}{\left(i_{2}, j_{1}\right)}, \frac{0.1776}{\left(i_{2}, j_{2}\right)}, \frac{60}{\left(\left(i_{2}, j_{3}\right)\right)}, \frac{60}{\left(i_{3}, j_{1}\right)}, \frac{60}{\left.i_{3}, j_{2}\right)}, \frac{60}{\left(i_{3}, j_{3}\right)}\right\} \\
& \hat{\mathbb{C}}\left(x_{2}\right)=\left\{\frac{60}{\left(i_{1}, j_{1}\right)}, \frac{0.0196}{\left(i_{1}, j_{2}\right)}, \frac{60}{\left(i_{1}, j_{3}\right)}, \frac{60}{\left(i_{2}, j_{1}\right)}, \frac{0.08208}{\left(i_{2}, j_{2}\right)}, \frac{60}{\left(\left(i_{2}, j_{3}\right)\right)}, \frac{60}{\left(i_{3}, j_{1}\right)}, \frac{60}{\left.i_{3}, j_{2}\right)}, \frac{60}{\left(i_{3}, j_{3}\right)}\right\}
\end{aligned}
$$

Table 9 Compute $\hat{\mathbb{C}}(x)(i, j)$

\begin{tabular}{llllll}
\hline$\hat{\mathbb{C}}$ & $\hat{\mathbb{C}}\left(x_{1}\right)$ & $\hat{\mathbb{C}}\left(x_{2}\right)$ & $\hat{\mathbb{C}}\left(x_{3}\right)$ & $\hat{\mathbb{C}}\left(x_{4}\right)$ & $\hat{\mathbb{C}}\left(x_{5}\right)$ \\
\hline$\left(i_{1}, j_{1}\right)$ & 60 & 60 & 60 & 60 & 60 \\
$\left(i_{1}, j_{2}\right)$ & 60 & 60 & 60 & 60 & 60 \\
$\left(i_{1}, j_{3}\right)$ & 60 & 60 & 60 & 60 & 60 \\
$\left(i_{2}, j_{1}\right)$ & 60 & 60 & 60 & 60 & 60 \\
$\left(i_{2}, j_{2}\right)$ & 0.1776 & 0.08208 & 0.0892 & 0.0632 & 0.083 \\
$\left(i_{2}, j_{3}\right)$ & 60 & 60 & 60 & 60 & 60 \\
$\left(i_{3}, j_{1}\right)$ & 60 & 60 & 60 & 60 & 60 \\
$\left(i_{3}, j_{2}\right)$ & 60 & 60 & 60 & 60 & 60 \\
$\left(i_{3}, j_{3}\right)$ & 60 & 60 & 60 & 60 & 60 \\
\hline
\end{tabular}




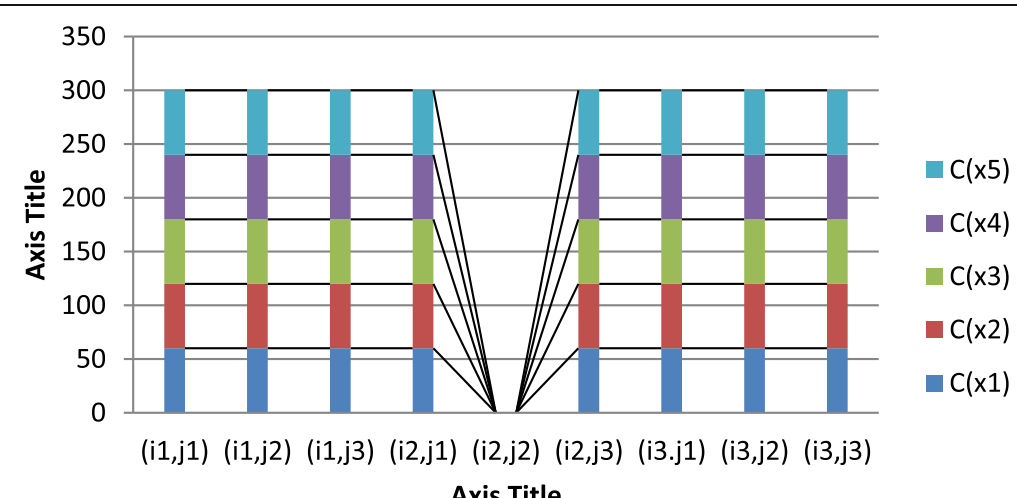

Fig. 9 Compute $\hat{\mathbb{C}}(x)(i, j)$. Compute the 3-polar fuzzy soft set $\hat{\mathbb{C}} \in\left([0,60]^{2 \times 3}\right)^{x}$, defined by $\hat{\mathbb{C}}(x)(i, j)=60 \wedge$ $\left.\sum_{k=1}^{3}\left(p_{k}{ }^{\circ} p_{1}{ }^{\circ} \mathbb{C}(x)(i, j) \times p_{k}{ }^{\circ} p_{2}{ }^{\circ} \mathbb{C}(x)(i, j)\right) \quad \forall(i, j) \in I^{2}, \forall x \in X\right)$ where $p_{k}:[0,60]^{2} \rightarrow[0,60]$ is the $k$-the projection $(k=1,2,3)$

$$
\begin{aligned}
& \hat{\mathbb{C}}\left(x_{3}\right)=\left\{\frac{60}{\left(i_{1}, j_{1}\right)}, \frac{60}{\left(i_{1}, j_{2}\right)}, \frac{60}{\left(i_{1}, j_{3}\right)}, \frac{60}{\left(i_{2}, j_{1}\right)}, \frac{0.0892}{\left(i_{2}, j_{2}\right)}, \frac{60}{\left(\left(i_{2}, j_{3}\right)\right)}, \frac{60}{\left(i_{3}, j_{1}\right)}, \frac{60}{\left.i_{3}, j_{2}\right)}, \frac{60}{\left(i_{3}, j_{3}\right)}\right\} \\
& \hat{\mathbb{C}}\left(x_{4}\right)=\left\{\frac{60}{\left(i_{1}, j_{1}\right)}, \frac{60}{\left(i_{1}, j_{2}\right)}, \frac{60}{\left(i_{1}, j_{3}\right)}, \frac{60}{\left(i_{2}, j_{1}\right)}, \frac{0.0632}{\left(i_{2}, j_{2}\right)}, \frac{60}{\left(\left(i_{2}, j_{3}\right)\right)}, \frac{60}{\left(i_{3}, j_{1}\right)}, \frac{60}{\left.i_{3}, j_{2}\right)}, \frac{60}{\left(i_{3}, j_{3}\right)}\right\} \\
& \hat{\mathbb{C}}\left(x_{5}\right)=\left\{\frac{60}{\left(i_{1}, j_{1}\right)}, \frac{60}{\left(i_{1}, j_{2}\right)}, \frac{60}{\left(i_{1}, j_{3}\right)}, \frac{60}{\left(i_{2}, j_{1}\right)}, \frac{0.083}{\left(i_{2}, j_{2}\right)}, \frac{60}{\left(\left(i_{2}, j_{3}\right)\right)}, \frac{60}{\left(i_{3}, j_{1}\right)}, \frac{60}{\left.i_{3}, j_{2}\right)}, \frac{60}{\left(i_{3}, j_{3}\right)}\right\}
\end{aligned}
$$

Now, we make a decision by two ways:

(1) First way:

Define a mapping $\mathbb{C}_{M}: X \rightarrow R$,by $\mathbb{C}_{M}(x)=\sum_{(i, j) \in I^{2}} \beta(x)(i, j)$, where

Table 10 Compute a mapping $\mathbb{C}_{M}: X \rightarrow R$, by $\mathbb{C}_{M}(x)=\sum_{(i, j) \in R^{2}} \beta(x)(i, j)$,

\begin{tabular}{llllll}
\hline$\hat{\mathbb{C}}$ & $\hat{\mathbb{C}}\left(x_{1}\right)$ & $\hat{\mathbb{C}}\left(x_{2}\right)$ & $\hat{\mathbb{C}}\left(x_{3}\right)$ & $\hat{\mathbb{C}}\left(x_{4}\right)$ & $\hat{\mathbb{C}}\left(x_{5}\right)$ \\
\hline$\left(i_{1}, j_{1}\right)$ & $\frac{60}{60}$ & 60 & 60 & 60 & 60 \\
$\left(i_{1}, j_{2}\right)$ & 60 & $\frac{60}{60}$ & 60 & 60 & 60 \\
$\left(i_{1}, j_{3}\right)$ & 60 & 60 & $\frac{60}{60}$ & 60 & 60 \\
$\left(i_{2}, j_{1}\right)$ & 60 & 0.08208 & 0.0892 & $\underline{60}$ & 60 \\
$\left(i_{2}, j_{2}\right)$ & $\underline{0.1776}$ & 60 & 60 & 0.0632 & 0.083 \\
$\left(i_{2}, j_{3}\right)$ & 60 & 60 & 60 & 60 & 60 \\
$\left(i_{3}, j_{1}\right)$ & $\underline{60}$ & $\underline{60}$ & 60 & 60 & 60 \\
$\left(i_{3}, j_{2}\right)$ & 60 & 60 & $\underline{60}$ & 60 & 60 \\
$\left(i_{3}, j_{3}\right)$ & 60 & 120.0 & 120.0 & 60 & 60 \\
$\mathbb{C}_{M}$ & 120.1776 & & & & 60 \\
\hline
\end{tabular}




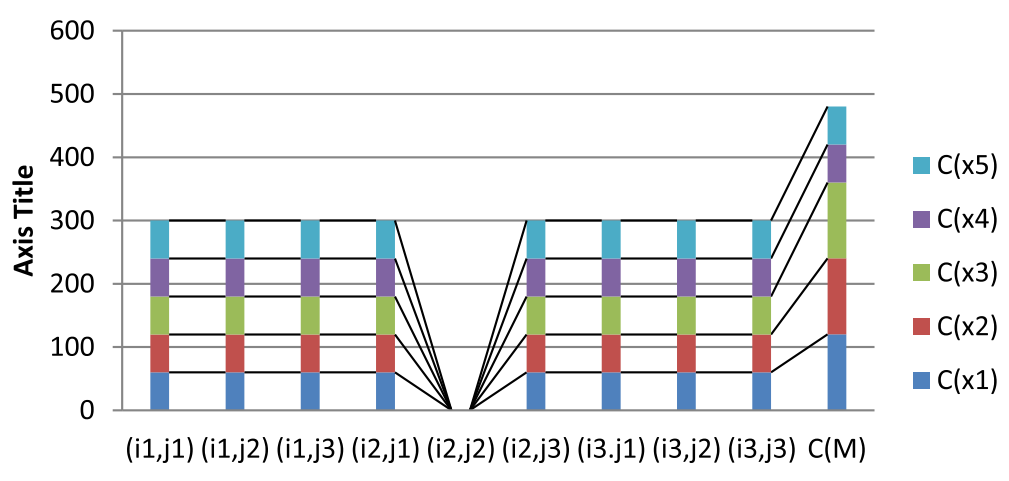

Axis Title

Fig. 10 Mapping $\mathbb{C}_{M}: X \rightarrow R$, by $\mathbb{C}_{M}(x)=\sum_{(i, j) \in I^{2}} \beta(x)(i, j)$. Define a mapping $\mathbb{C}_{M}: X \rightarrow R$, by $\mathbb{C}_{M}(x)=\sum_{(i, j) \in l^{2}} \beta(x)(i, j)$

$$
\beta(x)(i, j)=\left\{\begin{array}{cc}
\mathbb{C}(x)(i, j), & \mathbb{C}(x)(i, j)=\max \left\{\mathbb{C}(x)(s, t):(s, t) \in I^{2}\right\} \\
0 & \text { Otherwise }
\end{array}\right.
$$

From the following table; (in Table 10 and Fig. 10, we compute $\mathbb{C}_{M}: X \rightarrow R$, by $\mathbb{C}_{M}(x)$ $\left.=\sum_{(i, j) \in I^{2}} \beta(x)(i, j)\right)$

Since $\mathbb{C}_{M}\left(\left(x_{1}\right)=120.1776=\max \mathbb{C}_{M}\right.$, then the optimal alternative for the suitability of S-box to image encryption $\mathrm{X}$ based on 3-polar fuzzy soft set is $x_{1}$ (Residue encryption).

Motivated from the above problem, we give the following algorithm for decisionmaking problem:

Algorithm 5: Using 3-polar Fuzzy soft set.

Step 1. State $\mathcal{A}, \mathcal{B} \in\left[\left([0,60]^{2}\right)^{X} \times\left([0,60]^{2}\right)^{X}\right]^{I}=\left[\left([0,60]^{4}\right)^{X}\right]^{I}=\left[\left([0,60]^{4}\right)^{I}\right]^{X}=\left([0,60]^{4}\right)^{X \times I}$

Step 2. Compute $\mathbb{C}=\mathcal{A} \vee \mathcal{B} \in\left[\left([0,60]^{2}\right)^{X} \times\left([0,60]^{2}\right)^{X}\right]^{I}$

Step 3. Compute the 3-polar Fuzzy soft set $\widehat{\mathbb{C}} \in\left([0,60]^{2 \times 3}\right)^{X}$, defined by

$$
\left.\widehat{\mathbb{C}}(x)(i, j)=60 \wedge \sum_{k=1}^{3}\left(p_{k}{ }^{\circ} p_{1}{ }^{\circ} \mathbb{C}(x)(i, j) \times p_{k}{ }^{\circ} p_{2}{ }^{\circ} \mathbb{C}(x)(i, j)\right) \quad \forall(i, j) \in I^{2}, \forall x \in X\right)
$$

Where $p_{k}:[0,60]^{2} \rightarrow[0,60]$ is the $k$-the projection $(k=1,2,3)$;

Step 4. $\mathbb{C}_{M}: X \rightarrow R$, by $\mathbb{C}_{M}(x)=\sum_{(i, j) \in I^{2}} \beta(x)(i, j)$, where

$$
\beta(x)(i, j)=\left\{\begin{array}{cc}
\mathbb{C}(x)(i, j), & \mathbb{C}(x)(i, j)=\max \left\{\mathbb{C}(x)(s, t):(s, t) \in I^{2}\right\}, \\
0 & \text { 0therwise. }
\end{array}\right.
$$

Step 5. The maximal value of $\mathbb{C}_{M}$ to state the optimal alternative for suitability of S-box to image encryption based on 3-polar Fuzzy soft set.

(2) Second way: 
Table 11 Compute $m_{i}=\sum_{k=1}^{5}\left(x_{k}\right)(i, j), \quad x \in X, \quad(i, j) \in(I \times /)$ and compute $r_{i}=\sum_{j=1}^{9}\left(m_{i}-m_{j}\right)$ $(i=1,2,3,4,5)$

\begin{tabular}{|c|c|c|c|c|c|}
\hline$\hat{\mathbb{C}}$ & $\hat{\mathbb{C}}\left(x_{1}\right)$ & $\hat{\mathbb{C}}\left(x_{2}\right)$ & $\hat{\mathbb{C}}\left(x_{3}\right)$ & $\hat{\mathbb{C}}\left(x_{4}\right)$ & $\hat{\mathbb{C}}\left(x_{5}\right)$ \\
\hline$\overline{\left(i_{1}, j_{1}\right)}$ & $\underline{60}$ & 60 & 60 & 60 & 60 \\
\hline$\left(i_{1}, j_{2}\right)$ & 60 & $\underline{60}$ & 60 & 60 & 60 \\
\hline$\left(i_{1}, j_{3}\right)$ & 60 & 60 & $\underline{60}$ & 60 & 60 \\
\hline$\left(i_{2}, j_{1}\right)$ & 60 & 60 & 60 & $\underline{60}$ & 60 \\
\hline$\left(i_{2}, j_{2}\right)$ & $\underline{0.1776}$ & 0.08208 & 0.0892 & 0.0632 & 0.083 \\
\hline$\left(i_{2}, j_{3}\right)$ & 60 & 60 & 60 & 60 & $\underline{60}$ \\
\hline$\left(i_{3}, j_{1}\right)$ & $\underline{60}$ & 60 & 60 & 60 & 60 \\
\hline$\left(i_{3}, j_{2}\right)$ & 60 & $\underline{60}$ & 60 & 60 & 60 \\
\hline$\left(i_{3}, j_{3}\right)$ & 60 & 60 & $\underline{60}$ & 60 & 60 \\
\hline
\end{tabular}

Compute $m_{i}=\sum_{k=1}^{5}\left(x_{k}\right)(i, j), \quad x \in X, \quad(i, j) \in(I \times I)$ and compute $r_{i}=\sum_{j=1}^{9}\left(m_{i}-m_{j}\right)$ $(i=1,2,3,4,5)$, then, (in Table 11, and Fig. 11, we compute $m_{i}=\sum_{k=1}^{5}\left(x_{k}\right)(i, j), \quad x \in X$, $(i, j) \in(I \times I)$ and in Table 12 and Fig. 12, compute $\left.r_{i}=\sum_{j=1}^{9}\left(m_{i}-m_{j}\right)(i=1,2,3,4,5)\right)$. $r_{1}=\left(m_{1}-m_{1}\right)+\left(\mathrm{m}_{1}-m_{2}\right)+\left(m_{1}-m_{3}\right)+\left(m_{1}-m_{4}\right)+\left(m_{1}-m_{5}\right)=(480.1776-480.1776)$ $+(480.1776-480.08208)+(480.1776-480.0892)+(480.1776-480.0632)+(480.1776-$ 480.083) $=0.39292$; similarity,

$r_{2}=-0.08468, r_{3}=-0.04908, r_{4}=-0.11968, r_{5}=-0.08008$. Since $r_{1}=0.39292=$ $\max r_{i}$, then the optimal alternative for the suitability of S-box to image encryption $\mathrm{X}$ based on 3-polar fuzzy soft set is $x_{1}$ (Residue encryption).

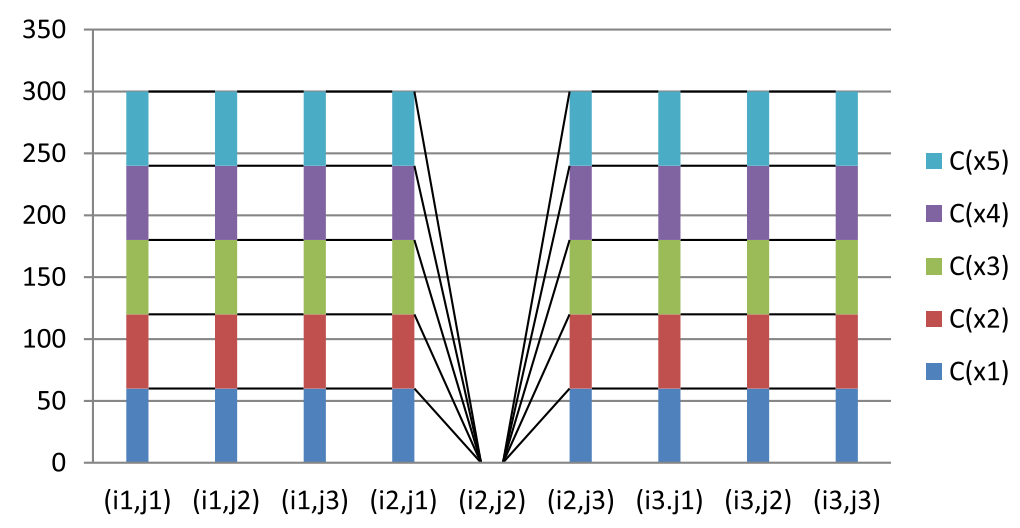

Fig. 11 Compute $m_{i}=\sum_{k=1}^{5}\left(x_{k}\right)(i, j), \quad x \in X, \quad(i, j) \in(I \times /)$ and compute $r_{i}=\sum_{j=1}^{9}\left(m_{i}-m_{j}\right) \quad(i=1,2,3,4,5)$. The maximal value of $\mathbb{C}_{\boldsymbol{M}}$ to state the optimal alternative for the suitability of S-box to image encryption based on 3-polar fuzzy soft set. (2) Second way:Compute $m_{i}=\sum_{k=1}^{5}\left(x_{k}\right)(i, j), \quad x \in X, \quad(i, j) \in(I \times I)$ and compute $r_{i}=\sum_{j=1}^{9}\left(m_{i}-m_{j}\right) \quad(i=1,2,3,4,5)$ 
Table 12 Compute $r_{i}=\sum_{j=1}^{3}\left(m_{i}-m_{j}\right) \quad(i, j=1,2,3,4,5)$

\begin{tabular}{lllllllllll}
\hline & $\left(i_{1}, j_{1}\right)$ & $\left(i_{1}, j_{2}\right)$ & $\left(i_{1}, j_{3}\right)$ & $\left(i_{2}, j_{1}\right)$ & $\left(i_{2}, j_{2}\right)$ & $\left(i_{2}, j_{3}\right)$ & $\left(i_{3}, j_{1}\right)$ & $\left(i_{3}, j_{2}\right)$ & $\left(i_{3}, j_{3}\right)$ & $m_{i}$ \\
\hline$x_{1}$ & 60 & 60 & 60 & 60 & 0.1776 & 60 & 60 & 60 & 60 & 480.1776 \\
$x_{2}$ & 60 & 60 & 60 & 60 & 0.08208 & 60 & 60 & 60 & 60 & 480.08208 \\
$x_{3}$ & 60 & 60 & 60 & 60 & 0.0892 & 60 & 60 & 60 & 60 & 480.0892 \\
$x_{4}$ & 60 & 60 & 60 & 60 & 0.0632 & 60 & 60 & 60 & 60 & 480.0632 \\
$x_{5}$ & 60 & 60 & 60 & 60 & 0.083 & 60 & 60 & 60 & 60 & 480.083 \\
\hline
\end{tabular}

Motivated from the above problem, we give the following algorithm for decisionmaking problem:

Algorithm 6: Using 3-polar Fuzzy soft set.

Step 1. State $\mathcal{A}, \mathcal{B} \in\left[\left([0,60]^{2}\right)^{X} \times\left([0,60]^{2}\right)^{X}\right]^{I}=\left[\left([0,60]^{4}\right)^{X}\right]^{I}=\left[\left([0,60]^{4}\right)^{I}\right]^{X}=\left([0,60]^{4}\right)^{X \times I}$

Step 2. Compute $\mathbb{C}=\mathcal{A} \wedge \mathcal{B} \in\left[\left([0,60]^{2}\right)^{X} \times\left([0,60]^{2}\right)^{X}\right]^{I}$

Step 3. Compute the 3-polar Fuzzy soft set $\widehat{\mathbb{C}} \in\left([0,60]^{2 \times 3}\right)^{X}$, defined by

$$
\left.\widehat{\mathbb{C}}(x)(i, j)=60 \wedge \sum_{k=1}^{3}\left(p_{k}{ }^{\circ} p_{1}{ }^{\circ} \mathbb{C}(x)(i, j) \times p_{k}{ }^{\circ} p_{2}{ }^{\circ} \mathbb{C}(x)(i, j)\right) \quad \forall(i, j) \in I^{2}, \forall x \in X\right)
$$

Where $p_{k}:[0,60]^{2} \rightarrow[0,60]$ is the $k$-the projection $(k=1,2,3)$;

Step 4. Compute $m_{i}=\sum_{k=1}^{5}\left(x_{k}\right)(i, j), x \in X,(i, j) \in(I \times I)$ and compute $r_{i}=\sum_{j=1}^{9}\left(m_{i}-\right.$ $\left.m_{j}\right)(i=1,2,3,4,5)$,

Step 5. The maximal value of $r_{i}=\sum_{j=1}^{3}\left(m_{i}-m_{j}\right) \quad(i, j=1,2,3,4,5)$ to state the optimal alternative for suitability of S-box to image encryption based on 3-polar Fuzzy soft set.

\section{Conclusion and remarks}

The major contributions in this paper can be summarized as follows:

1. We arrive to put a standard of optimal alternative for suitability of S-boxes to image encryption based on $m$-polar fuzzy soft set.

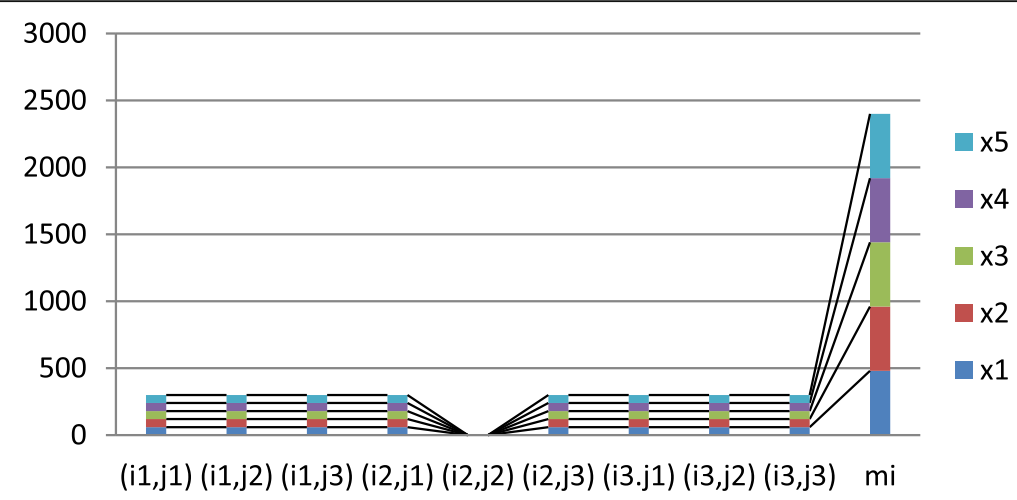

Fig. 12 Compute $r_{i}=\sum_{j=1}^{3}\left(m_{i}-m_{j}\right) \quad(i, j=1,2,3,4,5)$. The maximal value of $\mathbb{C}_{\boldsymbol{M}}$ to state the optimal alternative for the suitability of S-box to image encryption based on 3-polar fuzzy soft set. Should calculate $r_{i}=\sum_{j=1}^{3}\left(m_{i}-m_{j}\right) \quad(i, j=1,2,3,4,5)$ 
2. A novel design and model of real-life applications and explained in the existing literature the ways to the best choice of alternative for the suitability of S-boxes.

3. Studies the results of correlation analysis, entropy analysis, contrast analysis, homogeneity analysis, energy analysis, and mean of absolute deviation analysis of all S-boxes

4. The algorithm of the outcomes of these analyses is additionally observed and a $m$-polar fuzzy soft set decision-making criterion is used to decide the optimal alternative for suitability of S-box to image encryption applications.

In the future, we shall apply more advanced theories into

1. Interval-valued fuzzy soft sets in stochastic multi-criteria decision-making based on regret theory and prospect theory with combined weight;

2. Interval-valued fuzzy soft sets in emergency decision-making based on WDBA and CODAS with new information measure;

3. Hesitant fuzzy soft decision-making based on revised aggregation operators, WDBA and CODAS,

4. Pythagorean fuzzy set decision-making based on Pythagorean fuzzy set

Acknowledgements

The author is very appreciative to the reviewers for their precious comments which enormously ameliorated the quality of this paper.

Author's contributions

The author read and approved the final manuscript.

Funding

No place gives supporting or funding, only by the author.

Availability of data and materials

No data were used to support this study.

\section{Competing interests}

The author declares that there is no competing interests.

Received: 16 August 2019 Accepted: 1 January 2020

Published online: 26 March 2020

References

1. M. T. Tran, D.K. Buil, and A. D. Doung. Gray for S-box for advanced encryption standard," First International Conference on Computer Science Engineering and Applications 2008, 253-256

2. L. Zhang, X. Liao, and X. Wang, Neural information processing, 18th International Conference, ICONIP 2011, Shanghai, China, November 13-17, 2011, Proceedings, Part I

3. S. Y. Chen, W. C. Lin, and C.T. Chen, Graph. Models Imag. Proc. 1991, 453-457

4. Jing, F., Li, M., Zhang, H., Zhang, B.: Unsupervised image segmentation using local homogeneity analysis, - Proceedings of. ISCAS. 2, 456 (2003)

5. E. S. Gadelmawla, Nondestr. Test. Eval. 2004 Int. 37, 577

6. Avcibas, I., Memon, N., Sankur, B.: IEEE Trans. Imag. Proc. 212-221 (2003)

7. Prasadh, K., Ramar, K., Gnanajeyaraman, R.: Public key cryptosystems based on chaotic chebyshev polynomials. Journal of Engineering and Technology Research. 1(7), 122-128 (2009)

8. G. M. Alam, M. L. Kiah, B. B. Zaidan, A. A. Zaidan, and H. O. Alanazi, A new hybrid module ., Int International. Journal . Phys. Sci. 2010, 5: 3254-3260

9. R. Enayatifar, International Journal Physics Science 2011, 216- 221

10. J. Daemen and V. Rijmen, AES Proposal: Rijndael. AES Algorithm submission, Available: http://csrc.nist.gov/archive/aes/ rijndael/Rijndaelammended. pdf (1999).

11. Cui, L., Cao, Y.: International Journal Innov. Comput. I-3, 45 (2007)

12. J. Lui, B. Wai, X. Cheng, and X. Wang, Proc. International Conference on Computer Science information's network applications. (2005). 05, 724

13. Abuelyman, E.S., Alsehibani, A.A.S.: IJCSNS International. J. Compute. Sci. Network. Security. 8, 304-309 (2008)

14. I. Husain, T. Shah, and H. Mahmoud, A new algorithm to construct secure keys for AES Int. J. Contemp. Math. Sciences, Vol. 5, 2010, no. 26, $1263-1270$ 
15. Dueri, S., Maury, O.: Application of the APECOSM-E model to the skipjack tuna (Katsuwonus pelamis) fisheries of the Indian Ocean. Institute de Recherché pour le development, CRH Avenue Jean Monnet, France (1998)

16. Shi, X.Y., Xiao, H.U., You, X.C., Lam, K.Y.: American Journal of Applied Sciences. 8(8), 754-757 (2011)

17. Majumdar, P., Samanta, S.K.: Generalised fuzzy soft sets. Computers and Mathematics with Applications. 59, 1425-1432 (2010)

18. Pal, M., Dey, A.: Generalised multi-fuzzy soft set and its application in decision making. Pacific Science Review A: Natural Science and Engineering. 17, 23-28 (2015)

19. Agarwal, M., Biswas, K.K., Hanmandlu, M.: Generalized intuitionistic fuzzy soft sets with applications in decision- making. Applied Soft Computing. 13, 3552-3566 (2013)

20. Khalil, A.M.: Commentary on "Generalized intuitionistic fuzzy soft sets with applications in decision-making". Applied Soft Computing. 37, 519-520 (2015)

21. Hazaymeh, A.A., Abdullah, I.B., Balkhi, Z.T., Ibrahim, R.ı.: Generalized fuzzy soft expert set. Journal of Applied Mathematics. 22,328195 (2012)

22. Alkhazaleh, S., Salleh, A.R.: Generalised interval-valued fuzzy soft set. Journal of Applied Mathematics. 18, 870504 (2012)

23. Zhu, K., Zhan, J.: Fuzzy parameterized fuzzy soft sets and decision making. International Journal of Machine Learning and Cybernetics. 7, 1207-1212 (2016)

24. Zhao, H., Ma, W., Sun, B.: A novel decision making approach based on intuitionistic fuzzy soft sets. International Journal of Machine Learning and Cybernetics. 8, 1107-1117 (2017)

25. Deli, I.: Interval-valued neutrosophic soft sets and its decision making. International Journal of Machine Learning and Cybernetics. 8, 665-676 (2017)

26. Fatimah, F., Rosadi, D., Hakim, R.B.F., Alcantud, J.C.R.: N-soft sets and their decision making algorithms. Soft Computing 22(12), 3829-3842 (2018)

27. Fatimah, F., Rosadi, D., Hakim, R.B.F., Alcantud, J.C.R.: Probabilistic soft sets and dual probabilistic soft sets in decisionmaking. Neural Computing and Applications. 1-11 (2017). https://doi.org/10.1007/s00521-017-3011-y

28. Wang, H., Smarandache, F., Zhang, Y.Q., Sunderraman, R.: Single valued neutrosophic sets. Multispace Multistruct. 4, 410$413(2010)$

29. Ye, J.: Multicriteria decision-making method using the correlation coefficient under single-valued neutrosophic environment. International Journal of General Systems. 42, 386-394 (2013)

30. Ye, J.: Improved correlation coefficients of single valued neutrosophic sets and interval neutrosophic sets for multiple attribute decision making. Journal of Intelligent and Fuzzy Systems. 27, 2453-2462 (2014)

31. Ye, J.: Clustering methods using distance-based similarity measures of single-valued neutrosophic sets. Journal of Intelligent Systems. 23, 379-389 (2014)

32. Ye, J.: Multiple attribute group decision-making method with completely unknown weights based on similarity measures under single valued neutrosophic environment. Journal of Intelligent and Fuzzy Systems. 27, 2927-2935 (2014)

33. Peng, X.D., Dai, J.G.: Approaches to single-valued neutrosophic MADM based on MABAC, TOPSIS and new similarity measure with score function. Neural Computing and Applications. https://doi.org/10.1007/s00521-016-2607-y

34. Biswas, P., Pramanik, S., Giri, B.C.: TOPSIS method for multi-attribute group decision-making under single-valued neutrosophic environment. Neural Computing and Applications. 27, 727-737 (2016)

35. Sahin, R., Kucuk, A.: Subsethood measure for single valued neutrosophic sets. Journal of Intelligent and Fuzzy Systems. 29, 525-530 (2015)

36. Ghorabaee, M.K., Zavadskas, E.K., Olfat, L., Turskis, Z.: Multi-criteria inventory classification using a new method of evaluation based on distance from average solution (EDAS). Informatics. 26, 435-451 (2015)

37. S. Opricovic and G.H. Tzeng, Compromise solution by MCDM methods: a comparative analysis of VIKOR and TOPSIS, European Journal of Operational Research 156 (2004), 445-455.

38. Ghorabaee, M.K., Zavadskas, E.K., Amiri, M., Turskis, Z.: Extended EDAS method for fuzzy multi-criteria decision making: An application to supplier selection. International Journal of Computers Communications and Control. 11, 358-371 (2016)

39. R. S, Sahin and A. K."uc, "uk, On similarity and entropy of neutrosophic soft sets, Journal of Intelligent and Fuzzy Systems 27 (2014), 2417-2430.

40. Mukherjee, A., Sarkar, S.: Several similarity measures of neutrosophic soft sets and its application in real life problems. Annals of Pure and Applied Mathematics. 7, 1-6 (2014)

41. Fang, F., Jun, Y.B., Liu, X., Li, L.: An adjustable approach to fuzzy soft set based decision making. Journal of Computational and Applied Mathematics. 234, 10-20 (2010)

42. Liu, S.F., Dang, Y.G., Fang, Z.G.: Grey systems theory and its applications. Science Press, Beijing (2000)

43. Molodtsov, D.A.: Soft set theory-first results. Computers and Mathematics with Applications. 37, 19-31 (1999)

44. J. Chen, S.-G. Li, S.-Q. Ma and X. Wang, m-Polar fuzzy sets: An extension of bipolar fuzzy sets, The scientific world journal 2014: 416530

45. Koczy, L.T.: Vectorial I-fuzzy Sets. In: Gupta, M.M., Sanchez, E. (eds.) Approximate Reasoning in Decision Analysis, North Holland, Amsterdam, pp. 151-156 (1982) [45] Akram, M., m-Polar Fuzzy Graphs, Studies in Fuzziness and Soft Computing, Springer, 371(2019)

\section{Publisher's Note}

Springer Nature remains neutral with regard to jurisdictional claims in published maps and institutional affiliations. 